\title{
Supporting Information for Axially Chiral Guanidine as Highly Active and Enantioselective Catalyst for Electrophilic Amination of Unsymmetrically Substituted 1,3-Dicarbonyl Compounds
}

\author{
Masahiro Terada*, Megumi Nakano, and Hitoshi Ube \\ Graduate School of Science, Department of Chemistry, Tohoku University, Sendai 980-8578, Japan.
}

\begin{abstract}
General Information: Unless otherwise noted, the reactions were carried out with dried glassware under nitrogen atmosphere. Infrared spectra were recorded on a Shimazu FTIR-8600PC spectrometer. ${ }^{1} \mathrm{H}$ NMR spectra were recorded on a JEOL GSX-270 $(270 \mathrm{MHz})$ spectrometer. Chemical shifts are reported in ppm from the solvent resonance as the internal standard $\left(\mathrm{CDCl}_{3}: 7.26 \mathrm{ppm}\right)$. Data are reported as follows: chemical shift, integration, multiplicity ( $\mathrm{s}=$ singlet, $\mathrm{d}=$ doublet, $\mathrm{t}=$ triplet, $\mathrm{q}=$ quartet, sept $=$ septet, $\mathrm{b}=$ broad, $\mathrm{m}=$ multiplet), and coupling constants $(\mathrm{Hz}) .{ }^{13} \mathrm{C}$ NMR spectra were recorded on a JEOL GSX-270 (67.8 MHz) spectrometer with complete proton decoupling. Chemical shifts are reported in ppm from the solvent resonance as the internal standard $\left(\mathrm{CDCl}_{3}\right.$ : $77.0 \mathrm{ppm}$ ). Optically rotations were measured on a Jasco DIP-1000 digital polarimeter with a sodium lamp and reported as follows; $[\alpha]_{\lambda}{ }^{\mathrm{T}^{\circ} \mathrm{C}}(c=\mathrm{g} / 100 \mathrm{~mL}$, solvent). Analytical thin layer chromatography (TLC) was performed on Merck precoated TLC plates (silica gel $60 \mathrm{GF}_{254}, 0.25 \mathrm{~mm}$ ). Flash column chromatography was performed on silica gel 60N (spherical, neutral, 100-210 $\mu \mathrm{m}$; Kanto Chemical Co., Inc.). Mass spectra analysis was performed on a Bruker Daltonics APEX III FT-ICR-MS spectrometer at the Instrumental Analysis Center for Chemistry, Graduate School of Science, Tohoku University.
\end{abstract}

Matelials: Unless otherwise noted, materials were purchased from Wako Pure Chemical Industries, Ltd., Aldrich Inc., and other commercial suppliers and were used without purification. Following $\beta$-Keto esters (3) were prepared according to the literature procedures. ${ }^{1-3}$ All 1,3-dicarbonyl compounds were distilled prior to use. Dichloromethane, diethyl ether, tetrahydrofuran, and toluene were supplied from Kanto Chemical Co., Inc. as "Dehydrated solvent system". Other solvents were dried over activated MS4A and used under $\mathrm{N}_{2}$ atmosphere. 


\section{Experimental Section}

1. Representative Procedure for Preparation of Axially Chiral Guanidines (2)

Scheme 1. Synthesis of chiral guanidine 2a

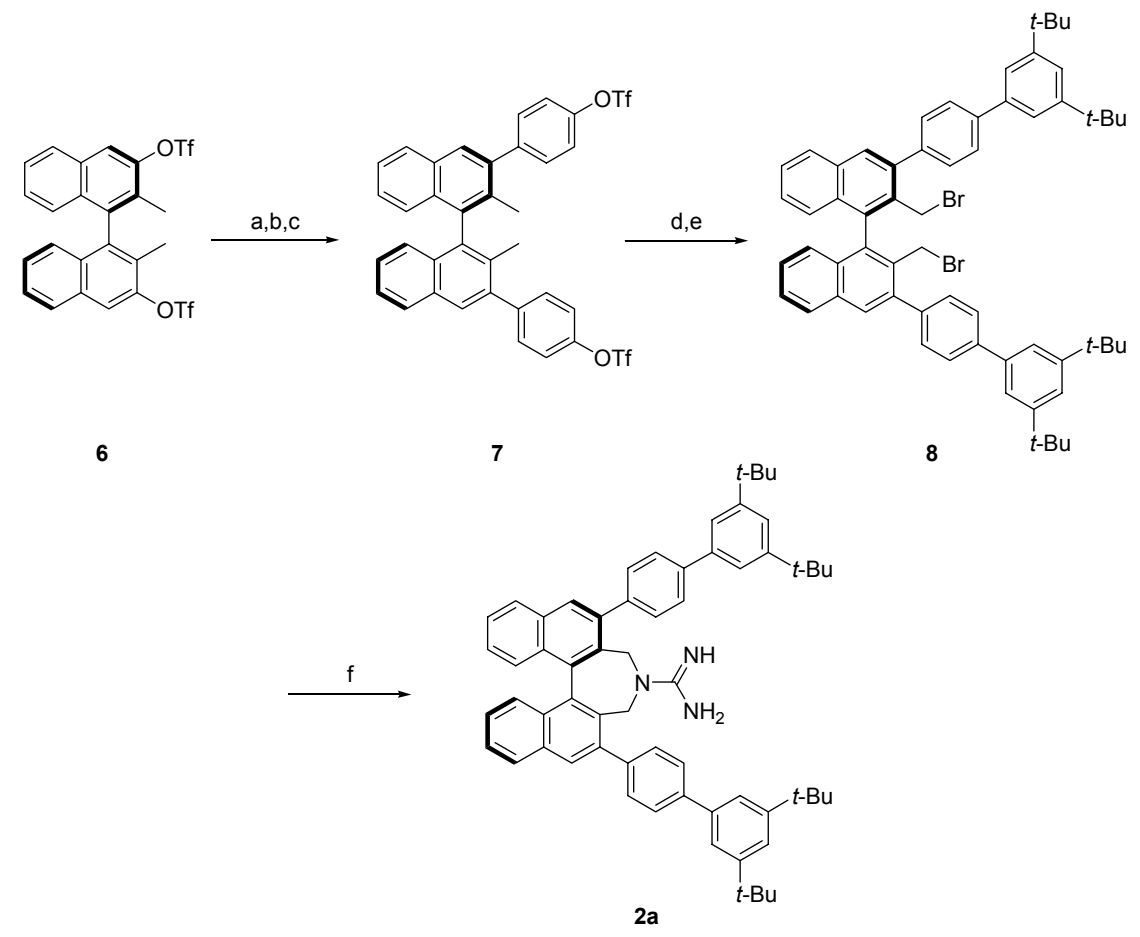

Reagents and conditions: (a) 4-MeO- $\mathrm{C}_{6} \mathrm{H}_{4} \mathrm{~B}(\mathrm{OH})_{2}, \mathrm{Pd}(\mathrm{OAc})_{2}, \mathrm{PPh}_{3}, \mathrm{~K}_{3} \mathrm{PO}_{4}, \mathrm{THF}, 65^{\circ} \mathrm{C}$; (b) $\mathrm{BBr}_{3}, \mathrm{CH}_{2} \mathrm{Cl}_{2}, 0{ }^{\circ} \mathrm{C}$; (c) $\mathrm{Tf}_{2} \mathrm{O}$, Py, Toluene, $0{ }^{\circ} \mathrm{C}$ to rt, $78 \%$ (3 steps); (d) 3,5- $t \mathrm{Bu}_{2}-\mathrm{C}_{6} \mathrm{H}_{3} \mathrm{~B}(\mathrm{OH})_{2}, \mathrm{Pd}(\mathrm{OAc})_{2}, \mathrm{PPh}_{3}, \mathrm{~K}_{3} \mathrm{PO}_{4}, \mathrm{THF}, 65^{\circ} \mathrm{C}$; (e) NBS, AIBN, benzene, $80{ }^{\circ} \mathrm{C}, 79 \%$ (2 steps); (f) guanidine, THF/EtOH $=1 / 3$, rt, $98 \%$<smiles></smiles>

(R)-3,3'-Bis(4-trifluoromethanesulfonyloxyphenyl)-2,2'-dimethyl-1,1'-binaphthyl (R)-7: A mixture of 6 (1.16g, 2 $\mathrm{mmol})$, prepared from $(R)$-binaphthol according to the literature procedure,${ }^{4} 4$-methoxyphenylboronic acid $(729 \mathrm{mg}$, $4.8 \mathrm{mmol}), \mathrm{Pd}(\mathrm{OAc})_{2}(22.5 \mathrm{mg}, 0.10 \mathrm{mmol})$, triphenylphosphine $(105 \mathrm{mg}, 0.40 \mathrm{mmol})$, and $\mathrm{K}_{3} \mathrm{PO}_{4}(1.71 \mathrm{~g}, 6.0 \mathrm{mmol})$ in THF $(6 \mathrm{~mL})$ was heated to $65^{\circ} \mathrm{C}$ and stirred for $15 \mathrm{~h}$ under $\mathrm{N}_{2}$ atmosphere. The resulting mixture was poured into saturated $\mathrm{NH}_{4} \mathrm{Cl}$ aq, and the whole mixture was filtered to remove the catalyst. The filtrate was extracted with ethyl acetate. The organic phase was washed with water and brine, dried over $\mathrm{Na}_{2} \mathrm{SO}_{4}$. After being concentrated and passed through short column chromatography, crude (R)-3,3'-Bis(4-methoxyphenyl)-1,1'-binaphthyl was obtained; ${ }^{1} \mathrm{H}$ NMR $\left(270 \mathrm{MHz}, \mathrm{CDCl}_{3}\right) \delta 1.95(6 \mathrm{H}, \mathrm{s}), 3.88(6 \mathrm{H}, \mathrm{s}), 7.00(4 \mathrm{H}, \mathrm{d}, J=6.8 \mathrm{~Hz}), 7.10(2 \mathrm{H}, \mathrm{d}, J=8.6 \mathrm{~Hz})$, $7.22(2 \mathrm{H}, \mathrm{d}, J=6.8,6.2 \mathrm{~Hz}), 7.39-7.44(6 \mathrm{H}, \mathrm{m}), 7.83(2 \mathrm{H}, \mathrm{s}), 7.88(2 \mathrm{H}, \mathrm{d}, J=8.6 \mathrm{~Hz})$. To a solution of 
bis(4-methoxyphenyl)-binaphthyl in $\mathrm{CH}_{2} \mathrm{Cl}_{2}(5 \mathrm{~mL})$ was added boron tribromide ( $\left.455 \mu \mathrm{L}, 4.8 \mathrm{mmol}\right)$ dropwise at 0 ${ }^{\circ} \mathrm{C}$. This mixture was stirred for $4 \mathrm{~h}$ at $0{ }^{\circ} \mathrm{C}$, and then water was added carefully. The organic layer was separated, and the aqueous phase was extracted with $\mathrm{CH}_{2} \mathrm{Cl}_{2}$. The organic extracts were washed with brine and dried over $\mathrm{Na}_{2} \mathrm{SO}_{4}$. After evaporation of the solvents, the crude (R)-3,3'-Bis(4-hydroxyphenyl)-2,2'-dimethyl-1,1'-binaphthyl was used for the following reaction without any purification. To a solution of Bis(4-hydroxyphenyl)-binaphthyl and pyridine $(485 \mu \mathrm{L}, 6.0 \mathrm{mmol})$ in toluene $(5 \mathrm{~mL})$ was added $\mathrm{Tf}_{2} \mathrm{O}(740 \mu \mathrm{L}, 4.4 \mathrm{mmol})$ dropwise at $0{ }^{\circ} \mathrm{C}$, and then the cooling bath was removed. The reaction mixture was stirred for $1 \mathrm{~h}$ at room temperature and poured into ice-cooled $1 N \mathrm{HCl}$ aq. The mixture was extracted with diethyl ether and washed with brine and then dried over $\mathrm{Na}_{2} \mathrm{SO}_{4}$. After being concentrated, the residue was purified by column chromatography (hexane $/ \mathrm{CH}_{2} \mathrm{Cl}_{2}=10 / 1$ to $4 / 1$ ) to give $(R)-7\left(1.15 \mathrm{~g}, 1.57 \mathrm{mmol}, 78 \%\right.$ yield (3 steps)); white solid; mp $209{ }^{\circ} \mathrm{C} ;[\alpha]_{\mathrm{D}}{ }^{32}+28.0\left(c 0.64, \mathrm{CHCl}_{3}\right) ;{ }^{1} \mathrm{H} \mathrm{NMR}(270$ $\left.\mathrm{MHz}, \mathrm{CDCl}_{3}\right) \delta 1.92(6 \mathrm{H}, \mathrm{s}), 7.09(2 \mathrm{H}, \mathrm{d}, J=8.4 \mathrm{~Hz}), 7.27(2 \mathrm{H}, \mathrm{dd}, J=8.4,7.0 \mathrm{~Hz}), 7.37(4 \mathrm{H}, \mathrm{d}, J=8.4 \mathrm{~Hz}), 7.45$ $(2 \mathrm{H}, \mathrm{dd}, J=7.8,7.0 \mathrm{~Hz}), 7.57(4 \mathrm{H}, \mathrm{d}, J=8.4 \mathrm{~Hz}), 7.84(2 \mathrm{H}, \mathrm{s}), 7.91(2 \mathrm{H}, \mathrm{d}, J=7.8 \mathrm{~Hz}) ;{ }^{13} \mathrm{C} \mathrm{NMR}(67.8 \mathrm{MHz}$, $\left.\mathrm{CDCl}_{3}\right) \delta 18.2,121.0,125.5,125.7,126.6,128.0,131.3,131.8,131.9,132.1,136.5,139.2,142.5,148.6$; IR (KBr): 3069, 2920, 2854, 1500, 1420, 1400, 1252, 1213, 1140, 1015, 883, 758, $611 \mathrm{~cm}^{-1}$; HRMS (ESI) Calcd for $\mathrm{C}_{36} \mathrm{H}_{24} \mathrm{~F}_{6} \mathrm{IO}_{6} \mathrm{~S}_{2}\left([\mathrm{M}+\mathrm{I}]^{-}\right)$856.9969. Found 856.9964.

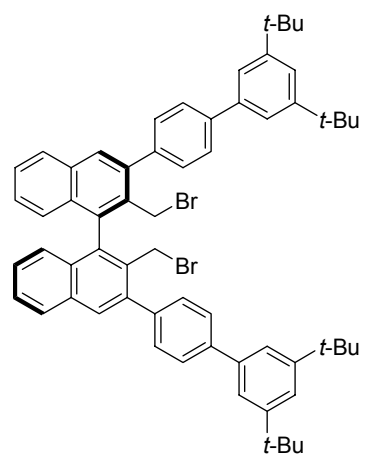

Dibromide (R)-8: A mixture of 7 (219 mg, $0.3 \mathrm{mmol})$, 3,5-di-tert-butylphenylboronic acid (210 $\mathrm{mg}, 0.90 \mathrm{mmol})$, $\mathrm{Pd}(\mathrm{OAc})_{2}(3.36 \mathrm{mg}, 0.015 \mathrm{mmol})$, triphenylphosphine $(15.7 \mathrm{mg}, 0.060 \mathrm{mmol})$, and $\mathrm{K}_{3} \mathrm{PO}_{4}(223 \mathrm{mg}, 1.05 \mathrm{mmol})$ in THF $(1.5 \mathrm{~mL})$ was heated to $65^{\circ} \mathrm{C}$ and stirred for $12 \mathrm{~h}$ under $\mathrm{N}_{2}$ atmosphere. The resulting mixture was poured into saturated $\mathrm{NH}_{4} \mathrm{Cl}$ aq, and the whole mixture was filtered. The filtrate was extracted with ethyl acetate and washed with brine. The organic phase was dried over $\mathrm{Na}_{2} \mathrm{SO}_{4}$. After being concentrated and passed short chromatography, crude $(R)$-3,3'-Bis(4-(3,5-di-tert-butylphenyl)phenyl)-2,2'-dimethyl-1,1'-binaphthyl was obtained; ${ }^{1} \mathrm{H}$ NMR (270 $\left.\mathrm{MHz}, \mathrm{CDCl}_{3}\right) \delta 1.42(36 \mathrm{H}, \mathrm{s}), 2.05(6 \mathrm{H}, \mathrm{s}), 7.16(2 \mathrm{H}, \mathrm{d}, J=8.3 \mathrm{~Hz}), 7.27(2 \mathrm{H}, \mathrm{ddd}, J=8.6,5.4,1.2 \mathrm{~Hz}), 7.43-47(6 \mathrm{H}$, m), $7.51(4 \mathrm{H}, \mathrm{d}, J=1.7 \mathrm{~Hz}), 7.58(4 \mathrm{H}, \mathrm{d}, J=8.3 \mathrm{~Hz}), 7.70(4 \mathrm{H}, \mathrm{d}, J=8.3 \mathrm{~Hz}), 7.92(2 \mathrm{H}, \mathrm{s}), 7.93(2 \mathrm{H}, \mathrm{d}, J=8.6 \mathrm{~Hz})$. The product was dissolved in benzene $(2 \mathrm{~mL}), N$-bromosuccinimide (NBS) (118 $\mathrm{mg}, 0.66 \mathrm{mmol})$ and 2,2'-azobis(isobutyronitrile) (AIBN) $(4.93 \mathrm{mg}, 0.030 \mathrm{mmol}$ ) was added. The mixture was heated and refluxed for 3 h. After being cooled to room temperature, this mixture was poured into water and extracted with diethyl ether. The organic extract was washed with brine and dried over $\mathrm{Na}_{2} \mathrm{SO}_{4}$ and concentrated. The residue was purified by column chromatography (hexane/AcOEt $=20 / 1$ as eluent) to give 8 (231 mg, $0.24 \mathrm{mmol}, 79 \%$ yield); white solid; mp $163{ }^{\circ} \mathrm{C}$ (decomp.); $[\alpha]_{\mathrm{D}}{ }^{34}-45.1\left(c 0.59, \mathrm{CHCl}_{3}\right) ;{ }^{1} \mathrm{H} \mathrm{NMR}\left(270 \mathrm{MHz}, \mathrm{CDCl}_{3}\right) \delta 1.41(36 \mathrm{H}, \mathrm{s}), 4.37(4 \mathrm{H}, \mathrm{s}), 7.20(2 \mathrm{H}$, 
d, $J=8.1 \mathrm{~Hz}), 7.34(2 \mathrm{H}, \mathrm{dd}, J=8.1,6.8 \mathrm{~Hz}), 7.47(2 \mathrm{H}, \mathrm{d}, J=1.9 \mathrm{~Hz}), 7.52-7.57(6 \mathrm{H}, \mathrm{m}), 7.73(8 \mathrm{H}, \mathrm{bs}), 7.94(2 \mathrm{H}, \mathrm{d}$, $J=8.1 \mathrm{~Hz}), 7.98(2 \mathrm{H}, \mathrm{s}) ;{ }^{13} \mathrm{C} \mathrm{NMR}\left(67.8 \mathrm{MHz}, \mathrm{CDCl}_{3}\right) \delta 31.7,35.2,121.7,121.8,126.7,127.4,127.5,128.1,130.0$, 130.7, 132.1, 132.6, 133.4, 136.6, 139.3, 140.3, 141.0, 141.8, 151.4; IR (KBr): 3055, 2963, 2903, 2864, 1595, 1508, $1474,1458,1393,1246,1217,1028,841,748,712 \mathrm{~cm}^{-1}$.

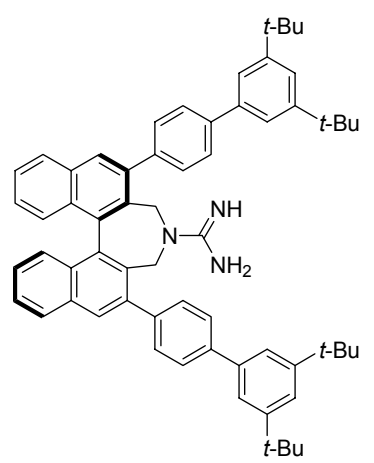

Axially chiral guanidine $(R)-2 \mathrm{a}\left(\mathrm{Ar}=p-\left(3,5-t-\mathrm{Bu}_{2} \mathrm{C}_{6} \mathrm{H}_{3}\right) \mathbf{C}_{6} \mathbf{H}_{4}-\right)$ : A solution of guanidinium chloride in ethanol was neutralized of Amberlite ${ }^{\circledR}$ IRA $400\left(\mathrm{OH}^{-}\right)$, and eluted with ethanol. After concentration in vacuo, free guanidine was dissolved in ethanol, and a solution of $8(287 \mathrm{mg}, 0.30 \mathrm{mmol})$ in THF $(2 \mathrm{~mL})$ was added, and the resulting mixture was stirred $12 \mathrm{~h}$ at room temperature. The reaction was quenched by $1 \mathrm{~N} \mathrm{HCl}$ aq and extracted with dichloromethane. The organic phase was dried over $\mathrm{Na}_{2} \mathrm{SO}_{4}$. After being concentrated, the guanidine hydrochloride salt was purified by column chromatography (Hexane/AcOEt $=4 / 1$ to AcOEt only). The guanidinium salt was neutralized by $2 \mathrm{~N}$ potassium hydroxide aq to give guanidine $2 \mathrm{a}(255 \mathrm{mg}, 0.29 \mathrm{mmol}, 98 \%)$; white solid; mp $213{ }^{\circ} \mathrm{C}$ (decomp.); $[\alpha]_{\mathrm{D}}{ }^{33}-44.0$ (c 0.60, $\left.\mathrm{CHCl}_{3}\right) ;{ }^{1} \mathrm{H}$ NMR $\left(270 \mathrm{MHz}, \mathrm{CDCl}_{3}\right) \delta 1.35(36 \mathrm{H}, \mathrm{s}), 2.18$ $(3 \mathrm{H}, \mathrm{s}), 3.71(2 \mathrm{H}, \mathrm{d}, J=12.7 \mathrm{~Hz}), 4.89(2 \mathrm{H}, \mathrm{d}, J=12.7 \mathrm{~Hz}), 7.33(2 \mathrm{H}, \mathrm{dd}, J=8.1,8.4 \mathrm{~Hz}), 7.46-7.59(14 \mathrm{H}, \mathrm{m}), 7.75$ $(4 \mathrm{H}, \mathrm{d}, J=8.4 \mathrm{~Hz}), 7.99(2 \mathrm{H}, \mathrm{d}, J=8.1 \mathrm{~Hz}), 8.04(2 \mathrm{H}, \mathrm{s}) ;{ }^{13} \mathrm{C} \mathrm{NMR}\left(67.8 \mathrm{MHz}, \mathrm{CDCl}_{3}\right) \delta 31.7,35.1,45.6,121.6$, $126.1,126.4,127.4,127.5,128.3,129.9,130.0,130.6,130.8,132.7,136.2,139.2,139.4,139.8,141.5,151.1$; IR (KBr): 3460, 3049, 2961, 2910, 2863, 1626, 1595, 1394, 1366, 1248, 1175, 1028, 895, 843, 746, $714 \mathrm{~cm}^{-1}$; HRMS (ESI) Calcd for $\mathrm{C}_{63} \mathrm{H}_{68} \mathrm{~N}_{3}\left([\mathrm{M}+\mathrm{H}]^{+}\right)$866.5408. Found 866.5411.

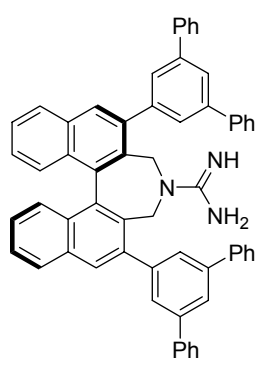

$(\boldsymbol{R})-\mathbf{2 b}\left(\mathbf{A r}=\mathbf{3 , 5}-\mathbf{P h}_{\mathbf{2}} \mathbf{C}_{\mathbf{6}} \mathbf{H}_{3}-\right)$ : White solid; $\mathrm{mp} 200{ }^{\circ} \mathrm{C}($ decomp. $) ;[\alpha]_{\mathrm{D}}{ }^{33}+103.5\left(c\right.$ 0.64, $\left.\mathrm{CHCl}_{3}\right) ;{ }^{1} \mathrm{H} \mathrm{NMR}(270 \mathrm{MHz}$ $\left.\mathrm{CDCl}_{3}\right) \delta 3.79(2 \mathrm{H}, \mathrm{d}, J=12.7 \mathrm{~Hz}), 4.91(2 \mathrm{H}, \mathrm{d}, J=12.7 \mathrm{~Hz}), 7.09(2 \mathrm{H}, \mathrm{d}, J=8.4 \mathrm{~Hz}), 7.28(2 \mathrm{H}, \mathrm{ddd}, J=8.4,7.0$, $1.6 \mathrm{~Hz}), 7.52(2 \mathrm{H}, \mathrm{dd}, J=8.4,7.0,1.1 \mathrm{~Hz}), 7.75(4 \mathrm{H}, \mathrm{d}, J=8.1 \mathrm{~Hz}), 7.83(4 \mathrm{H}, \mathrm{d}, J=8.1 \mathrm{~Hz}), 7.97(2 \mathrm{H}, \mathrm{s}), 8.01(2 \mathrm{H}$, d, $J=8.4 \mathrm{~Hz}) ;{ }^{13} \mathrm{C}$ NMR $\left(67.8 \mathrm{MHz}, \mathrm{CDCl}_{3}\right) \delta 45.9,48.9,125.2,126.3,126.6,127.2,127.5,127.6,128.3,128.8$, 130.2, 130.4, 130.7, 132.8, 136.2, 139.4, 140.5, 141.5, 142.0, 156.8; IR (KBr): 3452, 3047, 3024, 2925, 2854, 1629, 
1591, 1494, 1340, 1254, 1165, 1080, 1032, 887, 758, $698 \mathrm{~cm}^{-1}$; HRMS (ESI) Calcd for $\mathrm{C}_{59} \mathrm{H}_{44} \mathrm{~N}_{3}\left([\mathrm{M}+\mathrm{H}]^{+}\right) 794.3530$. Found 794.3529

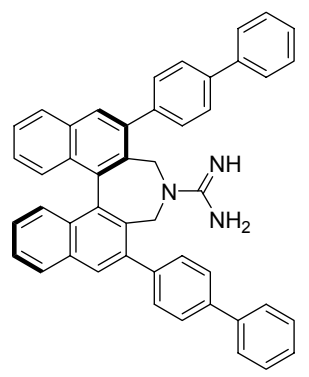

(R)-2c (Ar = p-Ph- $\left.\mathbf{C}_{6} \mathbf{H}_{4}-\right)$ : White solid; mp $178{ }^{\circ} \mathrm{C}$ (decomp.); $[\alpha]_{\mathrm{D}}{ }^{33}-11.0\left(c\right.$ 0.77, $\left.\mathrm{CHCl}_{3}\right) ;{ }^{1} \mathrm{H} \mathrm{NMR}(270 \mathrm{MHz}$ $\left.\mathrm{CDCl}_{3}\right) \delta 3.67(2 \mathrm{H}, \mathrm{d}, J=13.0 \mathrm{~Hz}), 4.80(2 \mathrm{H}, \mathrm{d}, J=13.0 \mathrm{~Hz}), 7.33(4 \mathrm{H}, \mathrm{m}), 7.40(4 \mathrm{H}, \mathrm{m}), 7.45-7.57(8 \mathrm{H}, \mathrm{m})$, 7.64-7.72 (8H, m), $7.98(2 \mathrm{H}, \mathrm{d}, J=8.3 \mathrm{~Hz}), 8.02(2 \mathrm{H}, \mathrm{s}, J=8.4 \mathrm{~Hz}) ;{ }^{13} \mathrm{C} \mathrm{NMR}\left(67.8 \mathrm{MHz}, \mathrm{CDCl}_{3}\right) \delta 45.4,126.2$, $126.5,126.8,127.0,127.2,127.4,127.5,128.3,128.7,130.0,130.1,130.2,130.6,132.8,136.2,139.2,139.3,140.2$, 140.3, 156.7; IR (KBr): 3458, 3063, 3024, 2966, 2935, 2854, 1643, 1591, 1491, 1240, 1168, 968, 895, 766, 735, 698 $\mathrm{cm}^{-1}$; HRMS (ESI) Calcd for $\mathrm{C}_{47} \mathrm{H}_{36} \mathrm{~N}_{3}\left([\mathrm{M}+\mathrm{H}]^{+}\right)$642.2904. Found 642.2901.

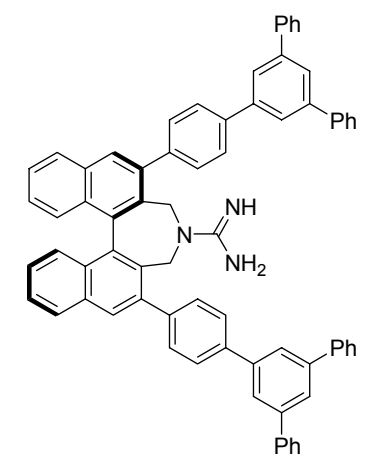

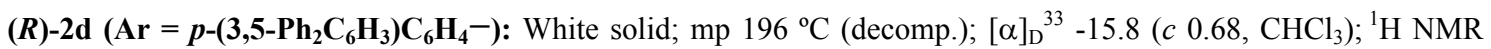
$\left(270 \mathrm{MHz} \mathrm{CDCl}_{3}\right) \delta 3.69(2 \mathrm{H}, \mathrm{d}, J=12.7 \mathrm{~Hz}), 4.87(2 \mathrm{H}, \mathrm{d}, J=12.7 \mathrm{~Hz}), 7.30-7.61(22 \mathrm{H}, \mathrm{m}), 7.72(2 \mathrm{H}, \mathrm{d}, J=7.6$ $\mathrm{Hz}), 7.79-7.85(6 \mathrm{H}, \mathrm{m}), 7.87(4 \mathrm{H}, \mathrm{s}), 7.99(2 \mathrm{H}, \mathrm{d}, J=8.1 \mathrm{~Hz}), 8.03(2 \mathrm{H}, \mathrm{s}) ;{ }^{13} \mathrm{C} \mathrm{NMR}\left(67.8 \mathrm{MHz}, \mathrm{CDCl}_{3}\right) \delta 45.6$, 125.0, 125.3, 126.1, 126.4, 127.3, 127.4, 127.5, 128.3, 128.8, 130.1, 130.2, 130.7, 130.8, 132.7, 136.2, 139.2,139.9, 140.1, 141.0, 141.5, 142.3, 157.5; IR (KBr): 3443, 3055, 3032, 2951, 2918, 2854, 1635, 1595, 1344, 1244, 1177 , 1080, 1032, 883, 843, 758, $698 \mathrm{~cm}^{-1}$; HRMS (ESI) Calcd for $\mathrm{C}_{71} \mathrm{H}_{52} \mathrm{~N}_{3}\left([\mathrm{M}+\mathrm{H}]^{+}\right)$946.4156. Found 946.4159. 
<smiles></smiles>

(R)-2e (Ar $=\boldsymbol{p}$-(3,5-( $\left.\left.\left(\mathbf{C F}_{3}\right)_{2} \mathbf{C}_{\mathbf{6}} \mathbf{H}_{3}\right) \mathbf{C}_{\mathbf{6}} \mathbf{H}_{4}-\right)$ : White solid; mp $199{ }^{\circ} \mathrm{C}($ decomp. $) ;[\alpha]_{\mathrm{D}}{ }^{33}+2.80\left(c 0.64, \mathrm{CHCl}_{3}\right) ;{ }^{1} \mathrm{H}$ NMR $\left(270 \mathrm{MHz} \mathrm{CDCl}_{3}\right) \delta 3.73(2 \mathrm{H}, \mathrm{d}, J=12.7 \mathrm{~Hz}), 4.83(2 \mathrm{H}, \mathrm{d}, J=12.7 \mathrm{~Hz}), 7.34(2 \mathrm{H}, \mathrm{dd}, J=8.1,7.0 \mathrm{~Hz}), 7.47(2 \mathrm{H}, \mathrm{d}$, $J=8.1 \mathrm{~Hz}), 7.56(2 \mathrm{H}, \mathrm{dd}, J=8.1,7.0 \mathrm{~Hz}), 7.62(4 \mathrm{H}, \mathrm{d}, J=8.1 \mathrm{~Hz}), 7.75(4 \mathrm{H}, \mathrm{d}, J=8.1 \mathrm{~Hz}), 7.88(2 \mathrm{H}, \mathrm{s}), 7.99(4 \mathrm{H}$, $\mathrm{d}, J=8.1 \mathrm{~Hz}), 8.10(4 \mathrm{H}, \mathrm{s}) ;{ }^{13} \mathrm{C} \mathrm{NMR}\left(67.8 \mathrm{MHz}, \mathrm{CDCl}_{3}\right) \delta 45.6,121.0\left(\mathrm{sept}, J_{C-F}=2.9 \mathrm{~Hz}\right), 123.5,125.3,126.4$, 126.6, 127.1, 127.2, 127.5, 128.3, 130.2, 130.4, 130.5, 130.7, 132.1 (q, $\left.J_{C-F}=33.4 \mathrm{~Hz}\right), 132.7,136.3,137.2,138.7$, 141.3, 142.5, 157.1; IR (KBr): 3443, 3055, 2926, 2862, 1649, 1624, 1589, 1381, 1277, 1177, 1132, 1057, 895, 835, 750, 698, $688 \mathrm{~cm}^{-1}$; HRMS (ESI) Calcd for $\mathrm{C}_{51} \mathrm{H}_{32} \mathrm{~F}_{12} \mathrm{~N}_{3}\left([\mathrm{M}+\mathrm{H}]^{+}\right)$914.2399. Found 914.2401.

\section{Confirmation of the 7 -membered ring structure of 2}

Scheme 2. Synthesis of chiral guanidine 2a from 7-membered ring amine 9

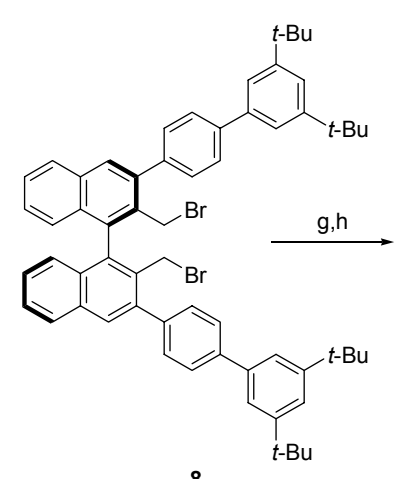

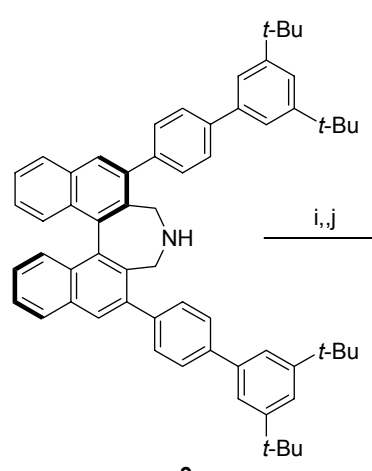

9

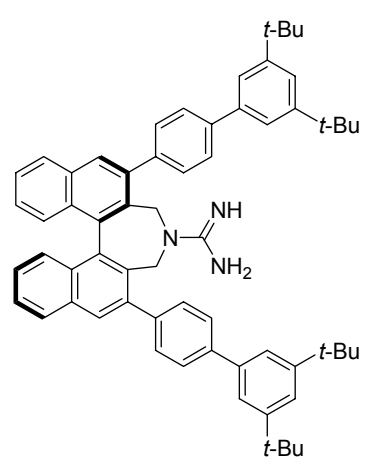

$2 a$

Reagents and conditions: (g) allylamine, THF, $60^{\circ} \mathrm{C}$; (h) $N, N$-dimethylbarbituric acid, $\mathrm{Pd}(\mathrm{OAc})_{2}, \mathrm{PPh}_{3}, \mathrm{CH}_{2} \mathrm{Cl}_{2}, 35^{\circ} \mathrm{C}$, $25 \%$ (2 steps); (i) $N, N$ '-bis(tert-butoxycarbonyl)-S-methylisothiourea, $\mathrm{CuI}, \mathrm{K}_{2} \mathrm{CO}_{3}, \mathrm{DMF}, 70{ }^{\circ} \mathrm{C}$; (j) $\mathrm{HCl}, \mathrm{MeOH}, 60$ ${ }^{\circ} \mathrm{C}, 26 \%$ (2 steps).<smiles></smiles> 
7-membered Amine (R)-9: To a solution of $8(194 \mathrm{mg}, 0.20 \mathrm{mmol})$ in THF $(4 \mathrm{~mL})$, was added allylamine $(45 \mu \mathrm{L}$, $0.60 \mathrm{mmol}$ ) at room temperature. The mixture was heated to $50{ }^{\circ} \mathrm{C}$, stirred for $24 \mathrm{~h}$, and then poured into water. After extraction with $\mathrm{CH}_{2} \mathrm{Cl}_{2}$, the organic extracts were dried over $\mathrm{Na}_{2} \mathrm{SO}_{4}$. After being concentrated and passed short column chromatography, the crude allyamine was dissolved in $\mathrm{CH}_{2} \mathrm{Cl}_{2}$ and $N, N$-dimethylbarbituric acid (93.7 $\mathrm{mg}, 0.60 \mathrm{mmol}), \mathrm{Pd}(\mathrm{OAc})_{2}(8.98 \mathrm{mg}, 0.040 \mathrm{mmol})$, triphenylphosphine $(42.0 \mathrm{mg}, 0.16 \mathrm{mmol})$ was added. The mixture was heated to $35^{\circ} \mathrm{C}$ and stirred overnight under nitrogen atmosphere. After cooling, $\mathrm{CH}_{2} \mathrm{Cl}_{2}$ was removed under vacuum and replaced by benzene. This mixture was washed twice with saturated $\mathrm{NaHCO}_{3}$ aq, dried over $\mathrm{Na}_{2} \mathrm{SO}_{4}$, and concentrated. The residue was purified by chromatography (AcOEt/Hex $=1 / 10$ as eluent) to give $(R)-9$ (42 mg, $0.051 \mathrm{mmol}, 25 \%$ yield (2 steps)); white solid; ${ }^{1} \mathrm{H}$ NMR $\left(270 \mathrm{MHz} \mathrm{CDCl}_{3}\right) \delta 1.43(36 \mathrm{H}, \mathrm{s}), 3.43(2 \mathrm{H}, \mathrm{d}, J=$ $12.4 \mathrm{~Hz}), 4.12(2 \mathrm{H}, \mathrm{d}, J=12.4 \mathrm{~Hz}), 7.29(2 \mathrm{H}, \mathrm{dd}, J=8.1,7.0 \mathrm{~Hz}), 7.45-7.53(10 \mathrm{H}, \mathrm{m}), 7.60-7.73(8 \mathrm{H}, \mathrm{m}), 7.97$ (2H, d, $J=8.1 \mathrm{~Hz}), 8.01(2 \mathrm{H}, \mathrm{s}) ;{ }^{13} \mathrm{C}$ NMR $\left(67.8 \mathrm{MHz}, \mathrm{CDCl}_{3}\right) \delta 31.6,35.1,121.4,121.6,125.9,127.2,127.5,128.2$, 129.9, 130.8, 132.5, 136.0, 139.4, 140.2, 141.3, 146.8, 151.1; IR (KBr): 3413, 2961, 2866, 1597, 1391, 1364, 1248, 1024, 891, 878, 835, 750, $711 \mathrm{~cm}^{-1}$; HRMS (ESI) Calcd for $\mathrm{C}_{62} \mathrm{H}_{65} \mathrm{~N}\left([\mathrm{M}+\mathrm{H}]^{+}\right) 824.5190$. Found 824.5193.

Guanidination of 7-membered ring amine: A mixture of 9 ( $37 \mathrm{mg}, 0.045 \mathrm{mmol}), \mathrm{CuI}(12 \mathrm{mg}, 0.063 \mathrm{mmol}), \mathrm{K}_{2} \mathrm{CO}_{3}$ (19 mg, $0.135 \mathrm{mmol}$ ) and $N, N^{\prime}$-bis(tert-butoxycarbonyl)-S-methylisothiourea (16 mg, $\left.0.054 \mathrm{mmol}\right)$ in DMF (450 $\left.\mu \mathrm{L}\right)$, was heated to $60{ }^{\circ} \mathrm{C}$ and stirred for overnight. The reaction mixture was poured into saturated $\mathrm{NH}_{4} \mathrm{Cl}$ and extracted with diethyl ether. The organic layer was washed with brine and dried over $\mathrm{Na}_{2} \mathrm{SO}_{4}$. After concentration and passed short column chromatography, the crude Boc protected guanidine was dissolved in $\mathrm{MeOH}(4 \mathrm{~mL})$ and conc $\mathrm{HCl}$ aq $(1 \mathrm{~mL})$ was added. The mixture was heated at $60{ }^{\circ} \mathrm{C}$ and stirred for $12 \mathrm{~h}$. The reaction mixture was added water and extracted with dichloromethane. The organic phase was dried over $\mathrm{Na}_{2} \mathrm{SO}_{4}$. After being concentrated, the guanidine hydrochloride salt was purified by column chromatography (Hexane/AcOEt $=4 / 1$ to AcOEt only). The guanidinium salt was neutralized by $2 \mathrm{~N}$ potassium hydroxide aq to give guanidine 2a $(10 \mathrm{mg}, 0.0115 \mathrm{mmol}, 2$ steps $26 \%$ ) as a white solid. ${ }^{1} \mathrm{H}$ and ${ }^{13} \mathrm{C}$ NMR spectra of guanidine $\mathbf{2 a}$ thus obtained agreed completely with those of $\mathbf{2 a}$ prepared from direct guanidination of $\mathbf{8}$ as shown in Scheme 1.

3. Representative Procedure for Enantioselective $\alpha$-amination of 1,3-Dicarbonyl Compounds (3) with Azodicarboxylate (4) Catalyzed by Axially Chiral Guanidine (2)

Reaction of Ethyl 2-oxocyclopentanecarboxylate (3a) with Di-tert-butyl Azodicarboxylate (4a) Catalyzed by 2a<smiles>CCOC(=O)C1CCCC1=O</smiles>

$3 a$

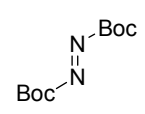

$4 a$

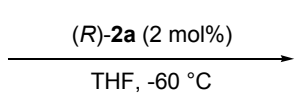

5 a

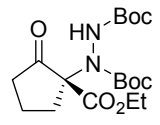

To a test tube equipped with a magnetic stirring bar was added THF $(1.0 \mathrm{~mL})$, ethyl 2-oxocyclopentanecarboxylate 3a $(16.3 \mu \mathrm{L}, 0.11 \mathrm{mmol})$ and $2 \mathrm{a}(1.73 \mathrm{mg}, 0.002 \mathrm{mmol})$. The tube was capped, and stirred at $\mathrm{rt}$ for 15 minutes. After that, the mixture was cooled to $-60{ }^{\circ} \mathrm{C}$ and di-tert-butyl azodicarboxylate (DBAD) 4a $(23.0 \mathrm{mg}, 0.10 \mathrm{mmol})$ was added. The resulting yellow solution was stirred until the yellow color of the solution was disappeared. After completion of the reaction, the resulting solution was quenched with saturated $\mathrm{NH}_{4} \mathrm{Cl}$ aq (a few drops), and solvents 
were removed. The residue was purified by column chromatography (hexane/AcOEt 10/1 to 4/1) to afford 5a in quantitative yield. Enantiomeric excess was determined by HPLC analysis.

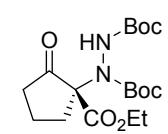

(R)-N'N-Bis(tert-butoxycarbonyl)-1-hydrazino-2-oxocyclopentanecarboxylic acid ethyl ester (5a): Colorless oil; $>99 \%$ yield; HPLC analysis Chiralpak AD-H (hexane/ $\mathrm{EtOH}=95 / 05,1.0 \mathrm{~mL} / \mathrm{min}, 210 \mathrm{~nm}, 10^{\circ} \mathrm{C}$ ) 7.4 (major), 9.9 $\min ; 97 \%$ ee; ${ }^{1} \mathrm{H}$ NMR $\left(\mathrm{CDCl}_{3}, 270 \mathrm{MHz}, \mathrm{rt}\right) \delta 1.28(3 \mathrm{H}, \mathrm{t}, J=7.0 \mathrm{~Hz}), 1.40-1.60(18 \mathrm{H}, \mathrm{m}), 1.73-2.60(6 \mathrm{H}, \mathrm{m}), 4.21$ $(2 \mathrm{H}, \mathrm{bq}), 6.51(1 \mathrm{H}, \mathrm{bs}) ;{ }^{13} \mathrm{C} \mathrm{NMR}\left(\mathrm{CDCl}_{3}, 67.8 \mathrm{MHz}, \mathrm{rt}\right) \delta 13.8,18.4,25.0,27.7,27.8,32.5,36.1,61.9,63.8,81.1$, 82.4, 154.1, 155.0, 167.8, 205.3; ${ }^{1} \mathrm{H}$ NMR $\left(\mathrm{CDCl}_{3}, 270 \mathrm{MHz}, 50{ }^{\circ} \mathrm{C}\right) \delta 1.27(3 \mathrm{H}, \mathrm{t}, J=7.0 \mathrm{~Hz}), 1.45(9 \mathrm{H}, \mathrm{s}), 1.46(9 \mathrm{H}$, s), 1.92-2.64 (6H, m), $4.22(2 \mathrm{H}, \mathrm{q} J=7.0 \mathrm{~Hz}), 6.46(1 \mathrm{H}, \mathrm{bs}) ;{ }^{13} \mathrm{C} \mathrm{NMR}\left(\mathrm{CDCl}_{3}, 67.8 \mathrm{MHz}, 50{ }^{\circ} \mathrm{C}\right) \delta 14.1,18.5,28.1$, 28.2, 28.3, 33.3, 36.8, 62.1, 81.4, 82.7, 154.2, 155.0, 167.8, 206.2; IR (neat): 3341, 2980, 2932, 1763, 1747, 1703, $1491,1369,1271,1240,1155,1103,1032,962,760 \mathrm{~cm}^{-1}$; HRMS (ESI) Calcd for $\mathrm{C}_{18} \mathrm{H}_{30} \mathrm{~N}_{2} \mathrm{NaO}_{7}\left([\mathrm{M}+\mathrm{Na}]^{+}\right)$ 409.1945. Found 409.1944; $[\alpha]_{\mathrm{D}}^{32}-3.47\left(c 1.09, \mathrm{CHCl}_{3}\right)$.

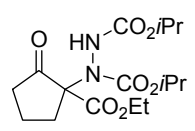

N'N-Bis(isopropyloxycarbonyl)-1-hydrazino-2-oxocyclopentanecarboxylic acid ethyl ester (5ab): Colorless oil; $>99 \%$ yield; HPLC analysis Chiralpak AD-H (Hexane/ EtOH =95/05, $1.0 \mathrm{~mL} / \mathrm{min}, 210 \mathrm{~nm}, 10{ }^{\circ} \mathrm{C}$ ) 11.9 (major), 16.7 $\min ; 89 \%$ ee; ${ }^{1} \mathrm{H}$ NMR $\left(\mathrm{CDCl}_{3}, 270 \mathrm{MHz}\right) \delta 1.21-1.29(15 \mathrm{H}, \mathrm{m}), 1.68-2.63(6 \mathrm{H}, \mathrm{m}), 4.21(2 \mathrm{H}, \mathrm{q}, J=6.8 \mathrm{~Hz}), 4.90$ $(2 \mathrm{H}$, septet, $J=5.9 \mathrm{~Hz}), 6.64(1 \mathrm{H}, \mathrm{m}) ;{ }^{13} \mathrm{C} \mathrm{NMR}\left(\mathrm{CDCl}_{3}, 67.8 \mathrm{MHz}\right) \delta 14.0,18.5,21.8,31.8,36.7,62.4,63.8,70.0$, 155.1, 155.8, 167.5, 206.3; IR (neat): 3261, 2986, 2937, 1767, 1732, 1707, 1508, 1381, 1250, 1153, 1111, 1020, 970, $781 \mathrm{~cm}^{-1}$; HRMS (ESI) Calcd for $\mathrm{C}_{16} \mathrm{H}_{26} \mathrm{~N}_{2} \mathrm{NaO}_{7}\left([\mathrm{M}+\mathrm{Na}]^{+}\right)$381.1632. Found 381.1633; $[\alpha]_{\mathrm{D}}{ }^{31}-0.32$ (c 0.99 , $\left.\mathrm{CHCl}_{3}\right)$.

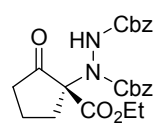

(R)- $N$ 'N-Bis(benzyloxycarbonyl)-1-hydrazino-2-oxocyclopentanecarboxylic acid ethyl ester (5ac): ${ }^{5}$ Colorless oil; $>99 \%$ yield; HPLC analysis Chiralpak AS-H (Hexane/iPrOH = 80/20, $1.0 \mathrm{~mL} / \mathrm{min}, 254 \mathrm{~nm}, 20^{\circ} \mathrm{C}$ ) 18.0 (major), $37.3 \mathrm{~min} ; 50 \%$ ee; ${ }^{1} \mathrm{H}$ NMR $\left(\mathrm{CDCl}_{3}, 270 \mathrm{MHz}\right) \delta 1.19$ (3H, bt), 1.81-2.69 (6H, m), 4.16 (2H, bq), 5.19 (4H, bs), 6.88 $(1 \mathrm{H}, \mathrm{bs}), 7.26-7.31(10 \mathrm{H}, \mathrm{m}) ;[\alpha]_{\mathrm{D}}^{31}+0.43\left(c 1.85, \mathrm{CHCl}_{3}\right),\left(\right.$ Lit. $^{2}:[\alpha]_{\mathrm{D}}+3.7(c 1.10),(99 \%$ ee, $(R)$-isomer $\left.)\right)$.<smiles>CCOC(=O)NC1(NC(=O)OC(C)(C)C)CCCCC1=O</smiles>

$N^{\prime} N$-Bis(tert-butoxycarbonyl)-1-hydrazino-2-oxocyclohexanecarboxylic acid ethyl ester (5b): ${ }^{6}$ Colorless oil; $>99 \%$ yield; HPLC analysis Chiralpak AD-H (hexane/ EtOH = 98/02, $1.0 \mathrm{~mL} / \mathrm{min}, 210 \mathrm{~nm}, 10^{\circ} \mathrm{C}$ ) 20.2 (major), 25.5 
$\min ; 98 \%$ ee; ${ }^{1} \mathrm{H}$ NMR $\left(\mathrm{CDCl}_{3}, 270 \mathrm{MHz}\right) \delta 1.27(3 \mathrm{H}, \mathrm{t}, J=7.0 \mathrm{~Hz}), 1.40-1.50(20 \mathrm{H}, \mathrm{m}), 1.67-3.20(6 \mathrm{H}, \mathrm{m}), 4.24$ $(2 \mathrm{H}, \mathrm{q}, J=7.0 \mathrm{~Hz}), 6.50(1 \mathrm{H}, \mathrm{bs}) ;[\alpha]_{\mathrm{D}}^{29}-24.1\left(c 0.41, \mathrm{CHCl}_{3}\right),\left(\mathrm{Lit} .:[\alpha]_{\mathrm{D}}-21.1\left(c 1.00, \mathrm{CHCl}_{3}\right),(83 \%\right.$ ee $\left.)\right)$.

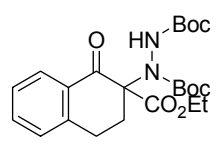

$N$ 'N-Bis(tert-butoxycarbonyl)-1-hydrazino-1,2,3,4-tetrahydro-1-oxonaphthalene-2-carboxylic acid ethyl ester (5c): Colorless oil; 99\% yield; HPLC analysis: Chiralpak AD-H, (Hexane/EtOH = 90/10, $1.0 \mathrm{~mL} / \mathrm{min}, 254 \mathrm{~nm}, 10$ $\left.{ }^{\circ} \mathrm{C}\right), 12.9,29.9$ (major) min; 97\% ee; ${ }^{1} \mathrm{H}$ NMR $\left(270 \mathrm{MHz}, \mathrm{CDCl}_{3}\right) \delta 1.18-1.47(21 \mathrm{H}, \mathrm{m}), 2.65-3.49(4 \mathrm{H}, \mathrm{m}), 4.30(2 \mathrm{H}$, q, $J=7.0 \mathrm{~Hz}), 6.36(1 \mathrm{H}, \mathrm{bs}), 7.24-7.28(2 \mathrm{H}, \mathrm{m}), 7.45(1 \mathrm{H}, \mathrm{bt}), 7.90(1 \mathrm{H}, \mathrm{dd}, J=7.0,7.0 \mathrm{~Hz}) ;{ }^{13} \mathrm{C} \mathrm{NMR}(67.8 \mathrm{MHz}$, $\left.\mathrm{CDCl}_{3}\right) \delta 13.9,14.1,25.5,27.8,31.0,61.9,62.2,80.9,82.7,126.4,127.7,128.4,131.6,133.4,154.4,155.5,169.4$, 190.9; IR (neat): 3298, 2983, 2935, 1744, 1707, 1701, 1364, 1300, 1252, 1157, 1057, 891, $743 \mathrm{~cm}^{-1}$; HRMS (ESI) Calcd for $\mathrm{C}_{25} \mathrm{H}_{36} \mathrm{~N}_{2} \mathrm{NaO}_{7}\left([\mathrm{M}+\mathrm{Na}]^{+}\right)$471.2102. Found 471.2103; $[\alpha]_{\mathrm{D}}{ }^{31}-24.6$ (c 2.36, $\left.\mathrm{CHCl}_{3}\right)$.

$$
\overbrace{\mathrm{CO}_{2} \mathrm{Et}}^{\mathrm{N}_{-\mathrm{Boc}}^{\mathrm{N}}}
$$

(S)-N'N-Bis(tert-butoxycarbonyl)-2-hydrazino-2-methyl-3-oxobutyric acid ethyl ester (5d): Colorless oil; >99\% yield; HPLC analysis: Chiralpak AD-H, (Hexane $/ \mathrm{iPrOH}=95 / 05,1.0 \mathrm{~mL} / \mathrm{min}, 210 \mathrm{~nm}, 10{ }^{\circ} \mathrm{C}$ ), 18.6 (major), 27.5 $\min ; 85 \%$ ee; ${ }^{1} \mathrm{H}$ NMR $\left(270 \mathrm{MHz}, \mathrm{CDCl}_{3}\right) \delta 1.29(3 \mathrm{H}, \mathrm{t}, J=7.0 \mathrm{~Hz}), 1.32-1.89(21 \mathrm{H}, \mathrm{m}), 2.36(3 \mathrm{H} . \mathrm{bs}), 4.23(2 \mathrm{H}, \mathrm{q}$, $J=7.0 \mathrm{~Hz}), 6.21(1 \mathrm{H}, \mathrm{m}) ;{ }^{13} \mathrm{C} \mathrm{NMR}\left(67.8 \mathrm{MHz}, \mathrm{CDCl}_{3}\right) \delta 14.1,19.1,19.9,24.3,28.0,28.1,62.0,81.3,82.8,84.7$, 155.4, 156.2, 169.2, 199.3; IR (neat): 3296, 2980, 2939, 2851, 1751, 1711, 1369, 1250, 1157, 1022, 829, $790 \mathrm{~cm}^{-1}$; HRMS (ESI) Calcd for $\mathrm{C}_{17} \mathrm{H}_{30} \mathrm{~N}_{2} \mathrm{NaO}_{7}\left([\mathrm{M}+\mathrm{Na}]^{+}\right)$397.1945. Found 397.1946; $[\alpha]_{\mathrm{D}}^{28}+23.1\left(c 0.71, \mathrm{CHCl}_{3}\right)$.

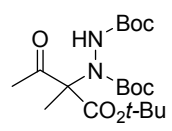

$N^{\prime} N$-Bis(tert-butoxycarbonyl)-2-hydrazino-2-methyl-3-oxobutyric acid tert-butyl ester (5e): Colorless oil; >99\% yield; HPLC analysis: Chiralpak AD-H, (Hexane $/ \mathrm{iPrOH}=95 / 05,1.0 \mathrm{~mL} / \mathrm{min}, 210 \mathrm{~nm}, 10^{\circ} \mathrm{C}$ ), 7.6 (major), $10.6 \mathrm{~min}$; $88 \%$ ee; ${ }^{1} \mathrm{H}$ NMR $\left(270 \mathrm{MHz}, \mathrm{CDCl}_{3}, \mathrm{rt}\right) \delta 1.21-1.65(30 \mathrm{H}, \mathrm{m}), 2.31(3 \mathrm{H}, \mathrm{bs}), 6.20(1 \mathrm{H}, \mathrm{m}) ;{ }^{13} \mathrm{C} \mathrm{NMR}(67.8 \mathrm{MHz}$, $\left.\mathrm{CDCl}_{3}, \mathrm{rt}\right) \delta 19.7,24.5,27.8,28.1,77.2,81.3,82.6,82.9,84.8,155.4,156.3,168.2,200.0 ;{ }^{1} \mathrm{H}$ NMR $(270 \mathrm{MHz}$, $\left.\mathrm{CDCl}_{3}, 50{ }^{\circ} \mathrm{C}\right) \delta 1.46(9 \mathrm{H}, \mathrm{s}), 1.49(9 \mathrm{H}, \mathrm{s}), 1.50(9 \mathrm{H}, \mathrm{s}), 2.35(3 \mathrm{H}, \mathrm{s}), 6.15(1 \mathrm{H}, \mathrm{bs}) ;{ }^{13} \mathrm{C} \mathrm{NMR}\left(67.8 \mathrm{MHz}, \mathrm{CDCl}_{3}, 50\right.$ $\left.{ }^{\circ} \mathrm{C}\right) \delta 19.7,24.7,28.0,28.1,28.3,81.3,82.6,155.2,155.4,167.9,200.2$; IR (neat): 3285, 2976, 2935, 2851, 1759, 1701, 1365, 1298, 1246, 1157, 1070, 843, $762 \mathrm{~cm}^{-1}$; HRMS (ESI) Calcd for $\mathrm{C}_{19} \mathrm{H}_{34} \mathrm{~N}_{2} \mathrm{NaO}_{7}\left([\mathrm{M}+\mathrm{Na}]^{+}\right) 425.2258$. Found 425.2256; $[\alpha]_{\mathrm{D}}{ }^{31}+26.5\left(c 0.73, \mathrm{CHCl}_{3}\right)$.

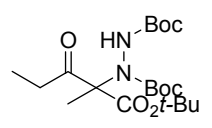

$N^{\prime} N$-Bis(tert-butoxycarbonyl)-2-hydrazino-2-methyl-3-oxopentanoic acid tert-butyl ester (5f): Colorless oil; 90\% yield; HPLC analysis: Chiralpak AD-H, (Hexane $/{ }^{i} \mathrm{PrOH}=96 / 04,1.0 \mathrm{~mL} / \mathrm{min}, 210 \mathrm{~nm}, 10{ }^{\circ} \mathrm{C}$ ), 6.9 (major), 9.9 
$\min ; 86 \%$ ee; ${ }^{1} \mathrm{H}$ NMR $\left(270 \mathrm{MHz}, \mathrm{CDCl}_{3}, \mathrm{rt}\right) \delta 1.44-1.82(32 \mathrm{H}, \mathrm{m}), 2.38-3.00(2 \mathrm{H}, \mathrm{bq}), 6.19(1 \mathrm{H}, \mathrm{bs}) ;{ }^{13} \mathrm{C} \mathrm{NMR}$ $\left(67.8 \mathrm{MHz}, \mathrm{CDCl}_{3}\right.$, rt) $\delta 8.4,15.3,20.2,27.8,28.0,28.129 .8,76.5,81.3,82.6,84.8,155.4,156.3,168.2,203.2 ;{ }^{1} \mathrm{H}$ NMR $\left(270 \mathrm{MHz}, \mathrm{CDCl}_{3}, 50{ }^{\circ} \mathrm{C}\right) \delta 1.09(\mathrm{bt}, 3 \mathrm{H}, J=6.8 \mathrm{~Hz}), 1.44(9 \mathrm{H}, \mathrm{s}), 1.57(18 \mathrm{H}, \mathrm{m}), 2.70(2 \mathrm{H}, \mathrm{bq}), 6.14(1 \mathrm{H}, \mathrm{bs})$; ${ }^{13} \mathrm{C}$ NMR $\left(67.8 \mathrm{MHz}, \mathrm{CDCl}_{3}, 50{ }^{\circ} \mathrm{C}\right) \delta 8.4,20.1,28.0,28.1,28.3,29.8,81.3,82.6,155.4,168.2,203.2$; IR (neat): $3337,2976,2943,1753,1701,1369,1248,1157,1130,1076,845,758 \mathrm{~cm}^{-1}$; HRMS (ESI) Calcd for $\mathrm{C}_{20} \mathrm{H}_{36} \mathrm{~N}_{2} \mathrm{NaO}_{7}$ $\left([\mathrm{M}+\mathrm{Na}]^{+}\right)$439.2415. Found 439.2417; $[\alpha]_{\mathrm{D}}^{27}+28.4\left(c 0.79, \mathrm{CHCl}_{3}\right)$.

$$
\overbrace{\mathrm{Et}}^{\mathrm{O}} \underset{\mathrm{CO}_{2}-\mathrm{Bu}}{\mathrm{HN}}
$$

$N$ 'N-Bis(tert-butoxycarbonyl)-2-hydrazino-2-ethyl-3-oxobutyric acid ethyl ester (5g): Colorless oil; 54\% yield; HPLC analysis: Chiralpak AD-H, (Hexane/ $\mathrm{PrOH}=95 / 05,1.0 \mathrm{~mL} / \mathrm{min}, 210 \mathrm{~nm}, 10{ }^{\circ} \mathrm{C}$ ), 6.2 (major), $9.2 \mathrm{~min} ; 62 \%$ ee; ${ }^{1} \mathrm{H}$ NMR $\left(270 \mathrm{MHz}, \mathrm{CDCl}_{3}\right) \delta 0.99(3 \mathrm{H}, \mathrm{t}, J=7.6 \mathrm{~Hz}), 1.41-1.46(27 \mathrm{H}, \mathrm{m}), 1.90-2.38(5 \mathrm{H}, \mathrm{m}), 6.19(1 \mathrm{H}, \mathrm{m}) ;{ }^{13} \mathrm{C}$ NMR (67.8 MHz, $\left.\mathrm{CDCl}_{3}\right) \delta 9.2,26.1,26.9,28.0,28.2,77.8,81.2,82.6,84.4,155.5,167.6,199.5$; IR (neat): 3339, 2981, 2935, 1751, 1707, 1475, 1394, 1369, 1252, 1157, 1020, 843, $762 \mathrm{~cm}^{-1}$; HRMS (ESI) Calcd for $\mathrm{C}_{20} \mathrm{H}_{36} \mathrm{~N}_{2} \mathrm{NaO}_{7}$ $\left([\mathrm{M}+\mathrm{Na}]^{+}\right)$439.2415. Found 439.2416; $[\alpha]_{\mathrm{D}}^{30}+5.23\left(c 0.20, \mathrm{CHCl}_{3}\right)$.

$$
\underbrace{\mathrm{N}}_{\mathrm{CO}_{2} t-\mathrm{Bu}}
$$

$N^{\prime} N$-Bis(tert-butoxycarbonyl)-2-hydrazino-2-formylpropionic acid tert-butyl ester (5h): Colorless oil; $>99 \%$ yield; HPLC analysis: Chiralpak AD-H, (Hexane/ $\mathrm{PrOH}=$ 98/02, $0.8 \mathrm{~mL} / \mathrm{min}, 210 \mathrm{~nm}, 10^{\circ} \mathrm{C}$ ), 11.9 (major), 14.9 $\min ; 83 \%$ ee; ${ }^{1} \mathrm{H}$ NMR $\left(270 \mathrm{MHz}, \mathrm{CDCl}_{3}\right) \delta 1.23-1.64(30 \mathrm{H}, \mathrm{m}), 6.32(1 \mathrm{H}, \mathrm{m}), 9.63(1 \mathrm{H}, \mathrm{bs}) ;{ }^{13} \mathrm{C}$ NMR $(67.8 \mathrm{MHz}$, $\left.\mathrm{CDCl}_{3}\right) \delta 17.3,27.9,28.0,28.1,77.2,81.5,82.9,83.2,155.1,192.5$; IR (neat): 3317, 2988, 2935, 1749, 1719, 1389 , 1356, 1244, 1153, 1136, $1072 \mathrm{~cm}^{-1}$; HRMS (ESI) Calcd for $\mathrm{C}_{18} \mathrm{H}_{32} \mathrm{~N}_{2} \mathrm{NaO}_{7}\left([\mathrm{M}+\mathrm{Na}]^{+}\right)$411.2102. Found 411.2104; $[\alpha]_{\mathrm{D}}^{27}-6.17\left(c 0.33, \mathrm{CHCl}_{3}\right)$.

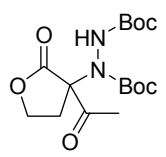

$N^{\prime} N$-Bis(tert-butoxycarbonyl)-2-hydrazino-2-acetyl- $\gamma$-butylolactone (5i): Colorless oil; $>99 \%$ yield; HPLC analysis Chiralpak AD-H (hexane/ $\mathrm{EtOH}=97 / 03,1.0 \mathrm{~mL} / \mathrm{min}, 210 \mathrm{~nm}, 10^{\circ} \mathrm{C}$ ) 21.6 (major), $30.0 \mathrm{~min} ; 15 \%$ ee; ${ }^{1} \mathrm{H}$ NMR $\left(\mathrm{CDCl}_{3}, 270 \mathrm{MHz}\right) \delta 1.40-1.44(18 \mathrm{H}, \mathrm{m}), 1.90-3.19(5 \mathrm{H}, \mathrm{m}), 4.33(2 \mathrm{H}, \mathrm{bt}), 6.88(1 \mathrm{H}, \mathrm{bs}) ;{ }^{13} \mathrm{C} \mathrm{NMR}\left(\mathrm{CDCl}_{3}\right.$, $67.8 \mathrm{MHz}) \delta 24.2,27.9,28.1,28.2,28.5,66.4,67.3,78.2,82.1,83.5,154.9,169.7,196.9$; IR (neat): 3348, 2984, $2935,1773,1744,1707,1356,1248,1153,1020,947,851,791 \mathrm{~cm}^{-1}$; HRMS (ESI) Calcd for $\mathrm{C}_{16} \mathrm{H}_{26} \mathrm{~N}_{2} \mathrm{NaO}_{7}$ $\left([\mathrm{M}+\mathrm{Na}]^{+}\right)$381.1632. Found 381.1633; $[\alpha]_{\mathrm{D}}^{28}-0.46\left(c 0.80, \mathrm{CHCl}_{3}\right)$.

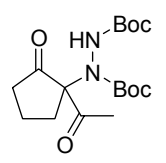


$N$ 'N-Bis(tert-butoxycarbonyl)-2-hydrazino-2-acetylcyclopentanone (5j): Colorless oil; >99\% yield; HPLC analysis Chiralpak AD-H (hexane/ $\mathrm{EtOH}=90 / 10,1.0 \mathrm{~mL} / \mathrm{min}, 210 \mathrm{~nm}, 10{ }^{\circ} \mathrm{C}$ ) 8.3 (major), $13.0 \mathrm{~min} ; 91 \%$ ee; ${ }^{1} \mathrm{H}$ $\operatorname{NMR}\left(\mathrm{CDCl}_{3}, 270 \mathrm{MHz}\right) \delta 1.40-1.42(18 \mathrm{H}, \mathrm{m}), 1.81-2.71(9 \mathrm{H}, \mathrm{m}), 6.54(1 \mathrm{H}, \mathrm{bs}) ;{ }^{13} \mathrm{C} \mathrm{NMR}\left(\mathrm{CDCl}_{3}, 67.8 \mathrm{MHz}\right) \delta$ 18.0, 24.9, 28.0, 28.1, 28.2, 37.1, 81.3, 81.7, 82.1, 155.2, 155.6, 167.8, 199.5, 209.2; IR (neat): 3329, 2982, 2935, $1751,1719,1369,1252,1155,1055,1018,887,766 \mathrm{~cm}^{-1}$; HRMS (ESI) Calcd for $\mathrm{C}_{17} \mathrm{H}_{28} \mathrm{~N}_{2} \mathrm{NaO}_{6}\left([\mathrm{M}+\mathrm{Na}]^{+}\right)$ 379.1840. Found 381.1838; $[\alpha]_{\mathrm{D}}^{30}+178.4\left(c 0.43, \mathrm{CHCl}_{3}\right)$.

\section{Lowering the Load (0.05 mol\%) of Catalyst $2 \mathrm{a}$}

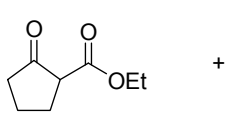

$3 \mathbf{a}$
$\mathrm{Boc}^{-11}{ }^{N-B o c}$

$4 \mathrm{a}$

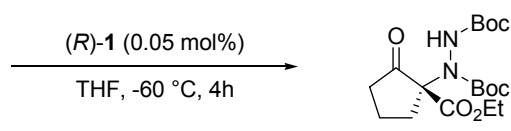

5a: quant, $97 \%$ ee

To a solution of ethyl 2-oxocyclopentanecarboxylate 3a $(490 \mu \mathrm{L}, 3.3 \mathrm{mmol})$ and 2a (1.30 $\mathrm{mg}, 0.0015 \mathrm{mmol})$ in THF $(10 \mathrm{~mL})$ was added di-tert-butyl azodicarboxylate $(691 \mathrm{mg}, 3.0 \mathrm{mmol})$ at $-60{ }^{\circ} \mathrm{C}$, and the resulting mixture was stirred for $4 \mathrm{~h}$. The reaction mixture was quenched by saturated $\mathrm{NH}_{4} \mathrm{Cl}$ aq and extracted by ethyl acetate. The organic phase was washed with brine, and dried over anhydrous $\mathrm{Na}_{2} \mathrm{SO}_{4}$. After removal of solvents, the residue was purified by column chromatography (hexane/AcOEt 10/1 as eluent) to afford 5a (1.16 g, >99\% yield, 97\% ee).

\section{Determination of Absolute Configuration of Cyclic Keto Ester (5a)}

\section{(1) Derivatization of Boc Protected 5a to 11}

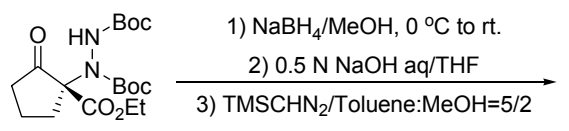

5 a $(97 \%$ ee)

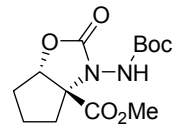

$10 \mathrm{a}$

3 steps, $66 \%$ yield
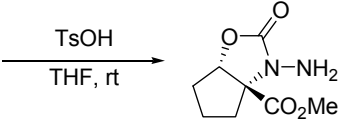

11

$89 \%$ yield

5a (3.0 mmol, 97\% ee) was dissolved in $10 \mathrm{~mL}$ of $\mathrm{MeOH}$. The solution was cooled to $0{ }^{\circ} \mathrm{C}$ and $\mathrm{NaBH}_{4}(113 \mathrm{mg}, 3.0$ mmol) solution in $\mathrm{MeOH}$ was added. After addition, the solution was allowed to warm to room temperature and stirred for 10 minutes. The resulting mixture was quenched by saturated $\mathrm{NH}_{4} \mathrm{Cl}$ aq and extracted by ethyl acetate and washed with brine. The organic phase was dried over $\mathrm{Na}_{2} \mathrm{SO}_{4}$. The residue was concentrated and $10 \mathrm{~mL}$ of THF and $10 \mathrm{~mL}$ of $0.5 \mathrm{~N} \mathrm{NaOH}$ aq was added. After stirring $12 \mathrm{~h}, 20 \mathrm{~mL}$ of ether was added to the reaction mixture and the diethyl ether fraction was back extracted with $0.5 \mathrm{~N} \mathrm{NaOH}$ aq. The aqueous phase was acidificated with $1 \mathrm{~N}$ $\mathrm{KHSO}_{4}$ aq and extracted with ether. The organic phase was dried over $\mathrm{Na}_{2} \mathrm{SO}_{4}$. Being concentrated, the residue was dissolved in $6 \mathrm{~mL}$ of $\mathrm{MeOH}$ and $15 \mathrm{~mL}$ of toluene and $2 \mathrm{~mL}$ of a $2 \mathrm{NTMSCHN}$ solution in diethyl ether was added drop wise. After 15 minutes excess of $\mathrm{TMSCHN}_{2}$ was decomposed by adding a few drops of acetic acid. After evaporation, the residue was purified by column chromatography (hexane/AcOEt 5/1 as eluent) to afford 10a (3 steps $66 \%$ yield); white solid; $\mathrm{mp} 84{ }^{\circ} \mathrm{C}$ (decomp.); ${ }^{1} \mathrm{H}$ NMR $\left(270 \mathrm{MHz}, \mathrm{CDCl}_{3}\right) \delta 1.48(9 \mathrm{H}, \mathrm{s}), 1.94-2.26(6 \mathrm{H}, \mathrm{m})$, $3.80(3 \mathrm{H}, \mathrm{s}), 4.87(2 \mathrm{H}, \mathrm{d}, J=5.7 \mathrm{~Hz}), 6.61(1 \mathrm{H}, \mathrm{bs}) ;{ }^{13} \mathrm{C} \mathrm{NMR}\left(67.8 \mathrm{MHz}, \mathrm{CDCl}_{3}\right) \delta 23.4,28.0,33.9,34.3,53.1,73.5$, 82.0, 82.9, 154.4, 154.8, 171.7; IR (KBr): 3294, 2984, 2951, 1774, 1740, 1521, 1392, 1369, 1273, 1252, 1167, 1150, 1107, 1026, 895, $750 \mathrm{~cm}^{-1}$; HRMS (ESI) Calcd for $\mathrm{C}_{18} \mathrm{H}_{13} \mathrm{~N}_{3} \mathrm{NaO}_{4}\left([\mathrm{M}+\mathrm{Na}]^{+}\right)$323.1214. Found 323.1215. 0.10 mmol of 10a $(30.0 \mathrm{mg})$ was dissolved in $1 \mathrm{~mL}$ of THF and cooled to $0{ }^{\circ} \mathrm{C}$. $\quad p$-Toluene sulfonic acid monohydrate 
(95.1 $\mathrm{mg}, 0.50 \mathrm{mmol}$ ) was added to the solution. After addition, the solution was allowed to warm to room temperature and stirred for 30 minutes. The resulting mixture was quenched by saturated $\mathrm{NaHCO}_{3}$ aq and extracted with ethyl acetate for 3 times. The organic phase was washed with brine and dried over $\mathrm{Na}_{2} \mathrm{SO}_{4} \quad \mathrm{After}$ evaporation, the residue was purified by column chromatography (hexane/AcOEt $2 / 1$ to $1 / 2$ ) to afford 11 (17.8 $\mathrm{mg}, 89 \%$ yield); Colorless oil; ${ }^{1} \mathrm{H}$ NMR $\left(\mathrm{CDCl}_{3}, 270 \mathrm{MHz}\right) \delta 1.68-2.35(6 \mathrm{H}, \mathrm{m}), 3.81(3 \mathrm{H}, \mathrm{s}), 3.99(2 \mathrm{H}, \mathrm{bs}), 4.80(1 \mathrm{H}, \mathrm{d}, J=5.9 \mathrm{~Hz})$; ${ }^{13} \mathrm{C} \mathrm{NMR}\left(\mathrm{CDCl}_{3}, 67.8 \mathrm{MHz}\right) \delta 23.3,32.9,34.4,53.0,74.1,82.4,157.4,171.8$; IR (neat): 3356, 2961, 2878, 1763, 1744, 1629, 1441, 1398, 1292, 1255, 1132, 1041, 991, 864, $768 \mathrm{~cm}^{-1}$; HRMS (ESI) Calcd for $\mathrm{C}_{18} \mathrm{H}_{13} \mathrm{~N}_{3} \mathrm{NaO}_{4}$ $\left([\mathrm{M}+\mathrm{Na}]^{+}\right)$223.0689. Found 223.0690; $[\alpha]_{\mathrm{D}}^{32}-20.5\left(c 0.80, \mathrm{CHCl}_{3}\right)$.

\section{(2) Derivatization of Stereochemically Known $(R)-5 \mathrm{ac}^{5}$ to $(R)-11$}

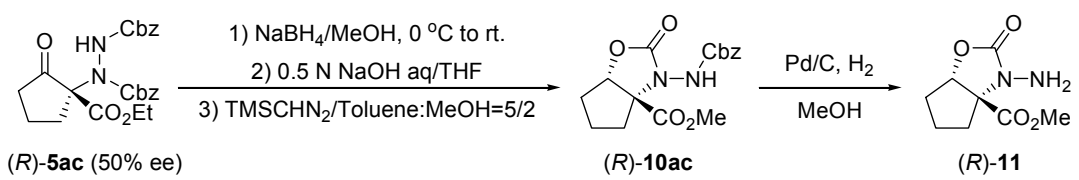

10ac was prepared from $(R)-\mathbf{5 a c}(50 \%$ ee) in a similar way of 10a. 10ac $(33.4 \mathrm{mg}, 0.20 \mathrm{mmol})$ was dissolved in a $\mathrm{MeOH}(2 \mathrm{~mL})$ and $5 \% \mathrm{Pd} / \mathrm{C}(20 \mathrm{mg})$ was added. The flask was filled with $\mathrm{H}_{2}$ and stirred for $4 \mathrm{~h}$ at room temperature. The reaction mixture was filtered through celite with $\mathrm{CH}_{2} \mathrm{Cl}_{2}$ and the solvent was evaporated. The residue was purified by column chromatography (hexane/AcOEt 2/1 to $1 / 2)$ to afford (R)-11 (19.0 mg, 95\% yield). $[\alpha]_{\mathrm{D}}^{32}-10.4\left(c 0.75, \mathrm{CHCl}_{3}\right)$. The absolute configuration of Boc protected $\mathbf{5 a}$ was determined to be $(R)$ by comparison of the optical rotation of $\mathbf{1 1 .}$

\section{Determination of Absolute Configuration of Acyclic Keto Ester (5d)}

\section{Derivatization of $5 d$ to Stereochemically Known $13^{5}$}

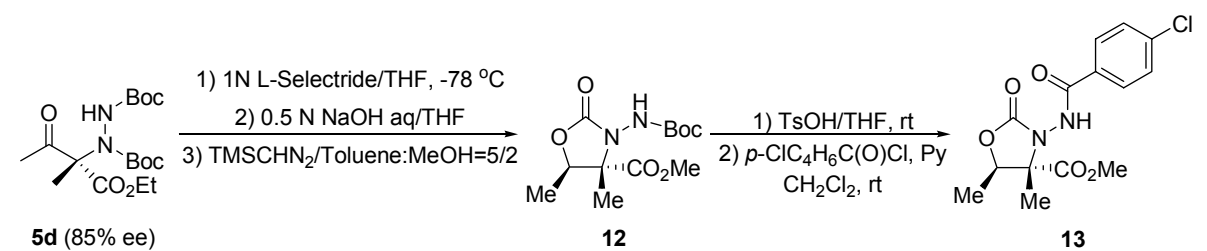

$\mathbf{1 2}$ was prepared from $\mathbf{5 d}$ according to the literature procedure ${ }^{5}$. Boc deprotection of $\mathbf{1 2}$ was performed as described before.

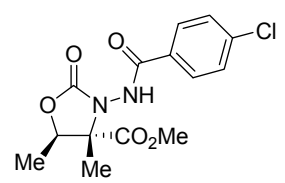

(4S,5R)-3-(4-Chloro-benzoylamino)-4,5-dimethyl-4-methoxycarbonyl-2-oxazolidinone (13): ${ }^{5}$ white solid; ${ }^{1} \mathrm{H}$ $\operatorname{NMR}\left(\mathrm{CDCl}_{3}, 270 \mathrm{MHz}\right) \delta 1.49(3 \mathrm{H}, \mathrm{s}), 1.56(3 \mathrm{H}, \mathrm{d}, J=6.6 \mathrm{~Hz}), 3.83(3 \mathrm{H}, \mathrm{s}), 4.87(1 \mathrm{H}, \mathrm{q}, J=6.6 \mathrm{~Hz}), 7.32(2 \mathrm{H}, \mathrm{d}$, $J=8.3 \mathrm{~Hz}), 7.69(2 \mathrm{H}, \mathrm{d}, J=8.3 \mathrm{~Hz}), 8.92(1 \mathrm{H}, \mathrm{bs}) ;{ }^{13} \mathrm{C} \mathrm{NMR}\left(\mathrm{CDCl}_{3}, 67.8 \mathrm{MHz}\right) \delta 1.1,15.4,16.0,53.2,67.8,129.0$, 129.3, 138.8, 155.2, 165.5, 172.8; $[\alpha]_{\mathrm{D}}^{32}-50.3\left(c 2.21, \mathrm{CHCl}_{3}\right)$. The absolute configuration of $\mathbf{5 d}$ was determined to be $(S)$ by comparison of the optical rotation of $\mathbf{1 3}$ with the following literature value: $\mathrm{Lit}^{5} .:[\alpha]_{\mathrm{D}}+39.5,(c 0.80),(98 \%$ ee, $(4 R, 5 S)$-isomer). 


\section{References}

1) Chini, M.; Crotti, P.; Macchia, F. J. Org. Chem. 1989, 54, 3930-3936.

2) Boyd, R. E.; Rasmussen, C. R.; Press, J. B. Synth. Commun. 1995, 27, 1045-1051.

3) Klingler, F. D.; Kreye, P.; Baumgarten, W. World Patent WO 2003087118 A2, 2003.

4) Ooi, T.; Kameda, M.; Maruoka, K. J. Am. Chem. Soc. 2003, 125, 5139-5151.

5) Marigo, M.; Juhl, K.; Jørgensen, K. A. Angew. Chem., Int. Ed. 2003, 42, 1367-1369.

6) Saaby, S.; Bella, M.; Jørgensen, K. A. J. Am. Chem. Soc. 2004, 126, 8120-8121. 

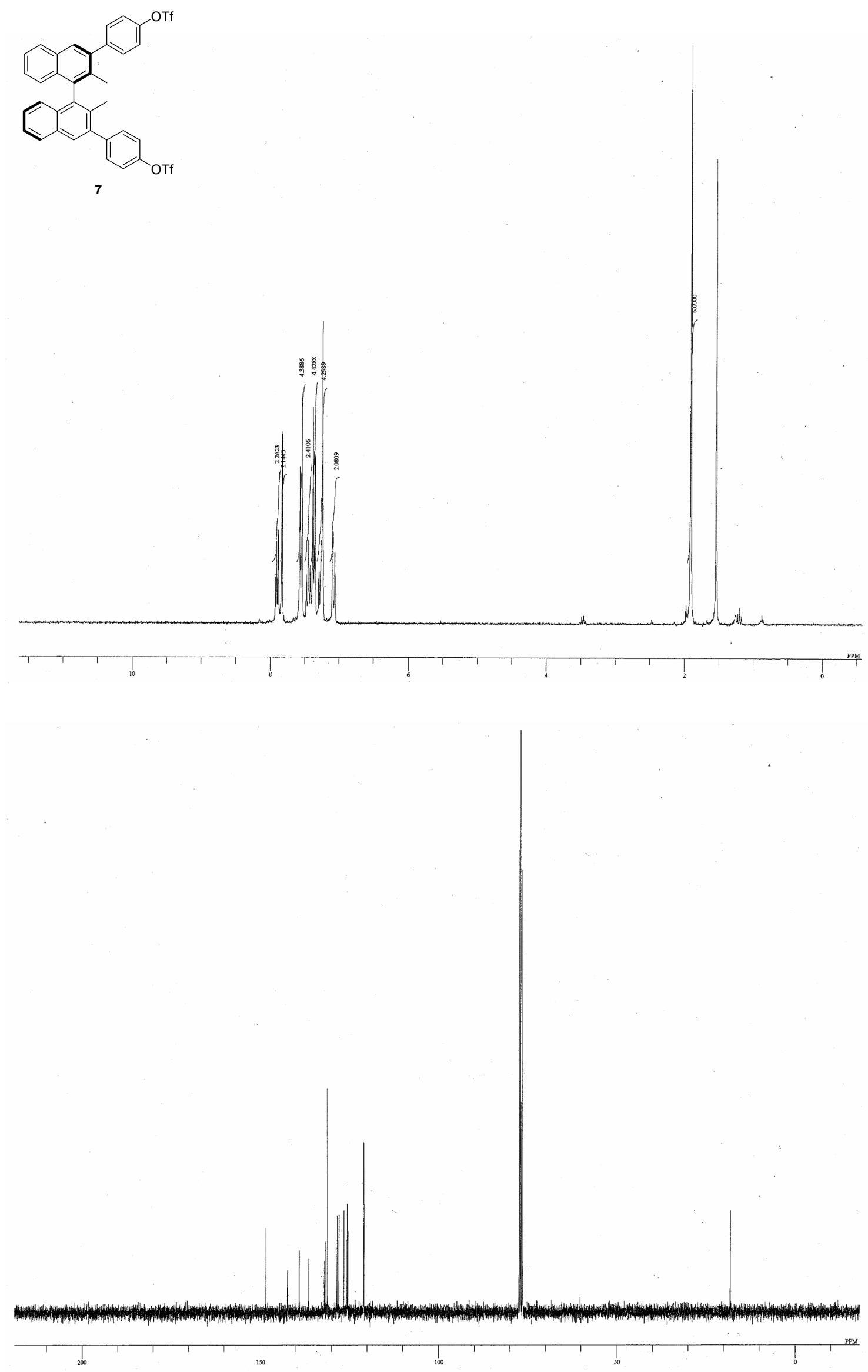

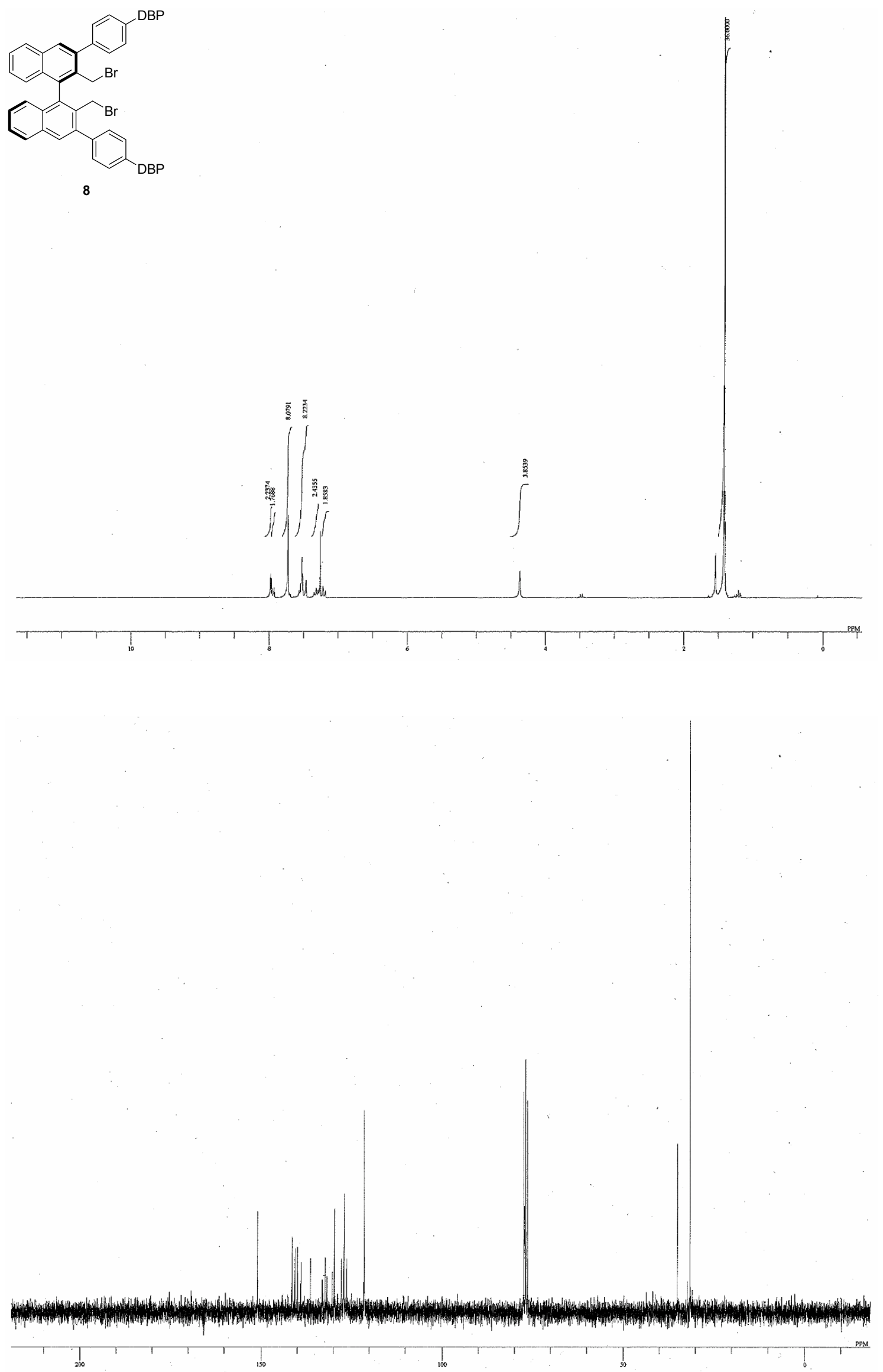

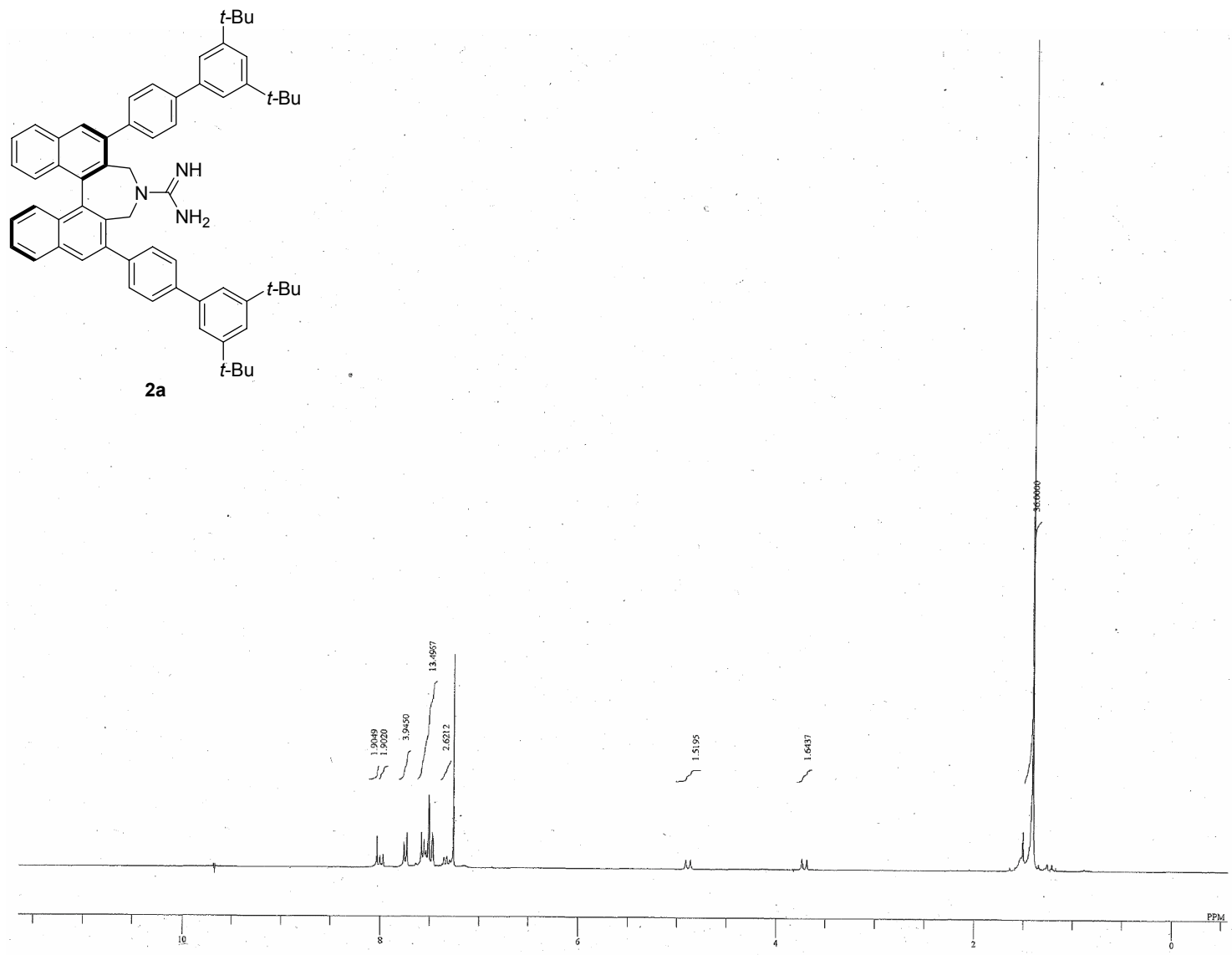

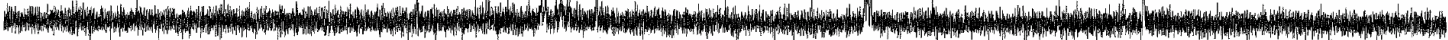




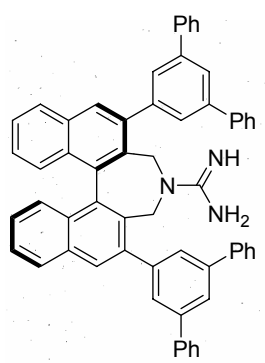

2b
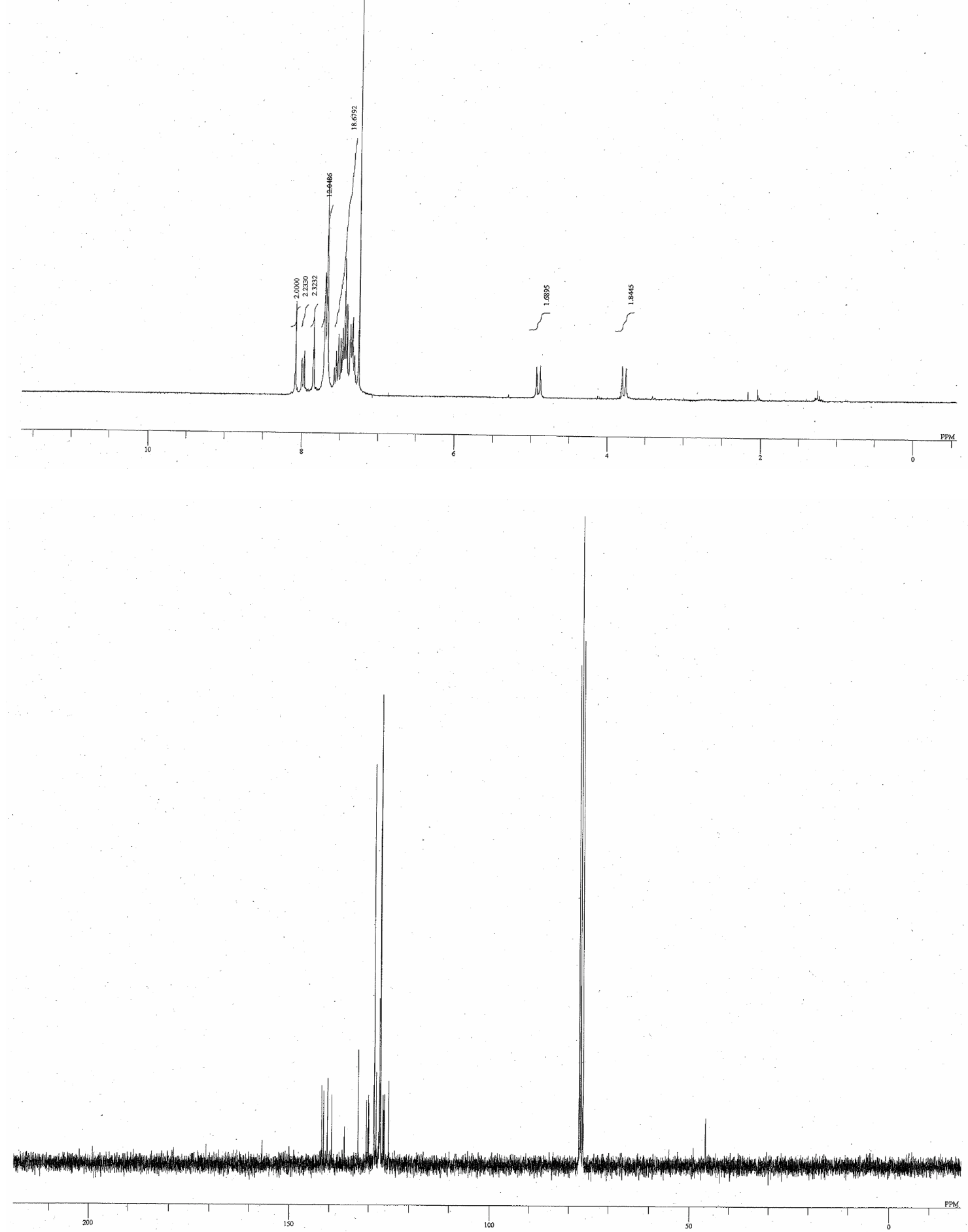


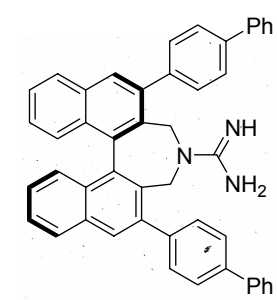

2c
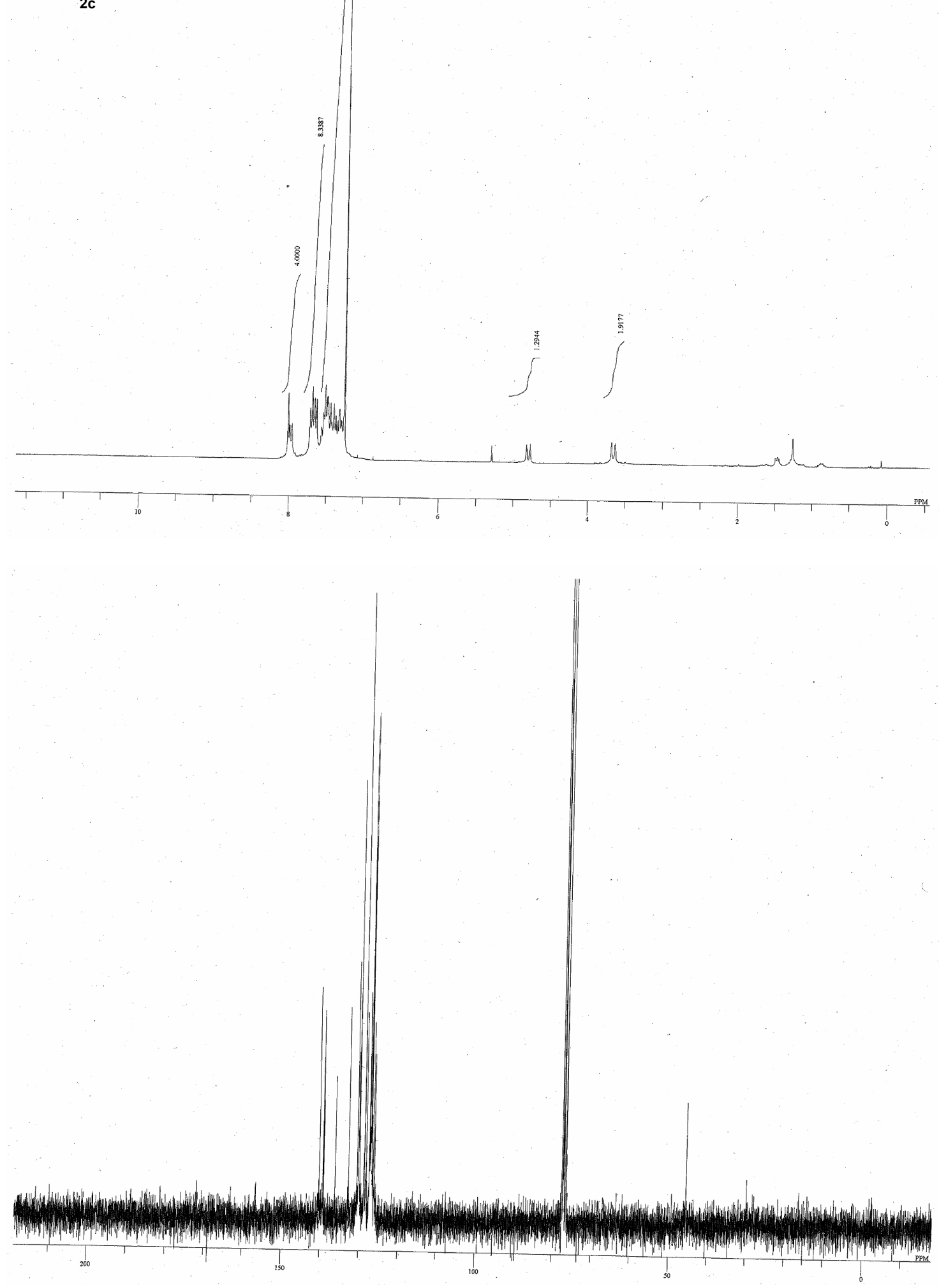

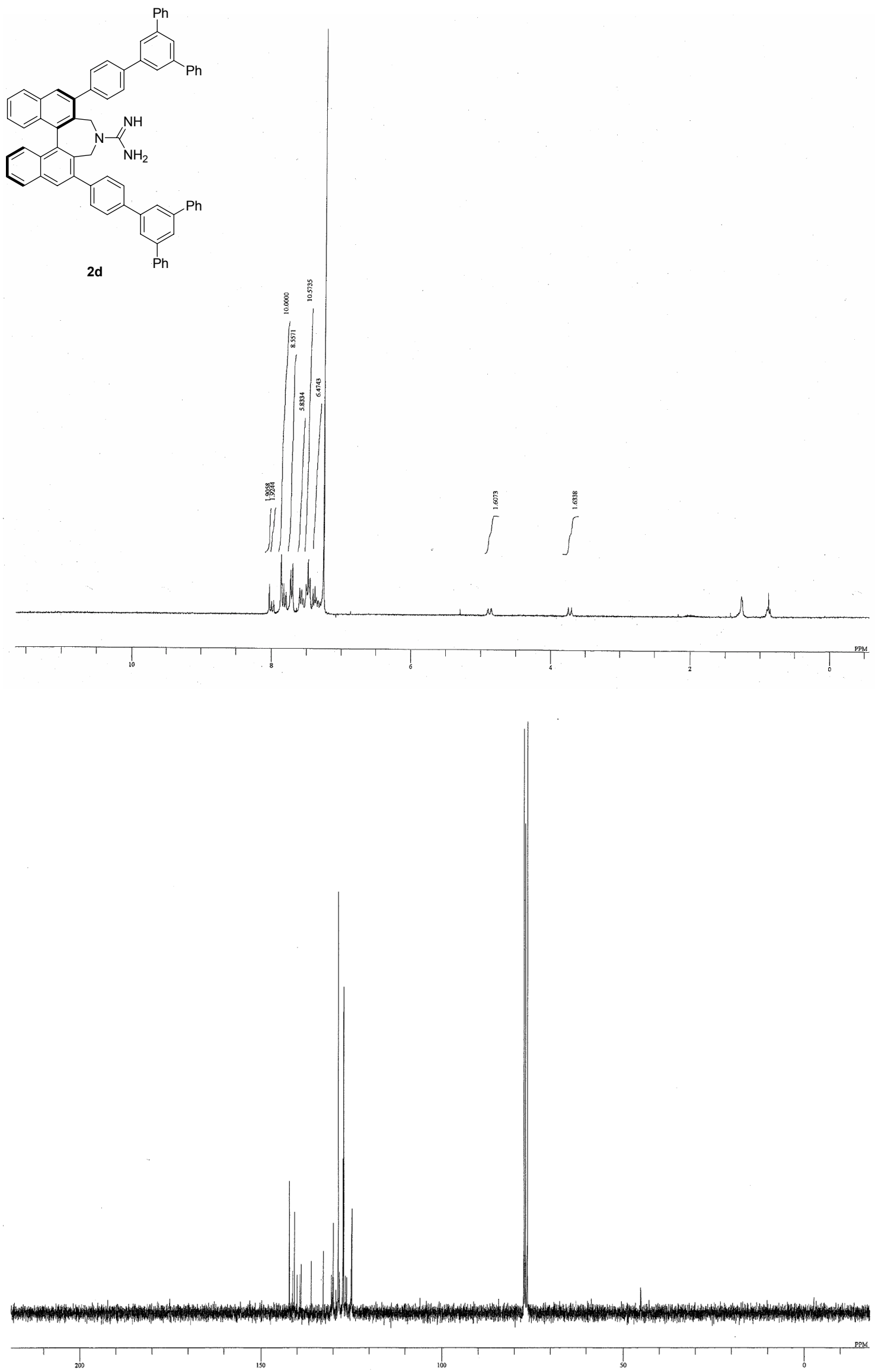

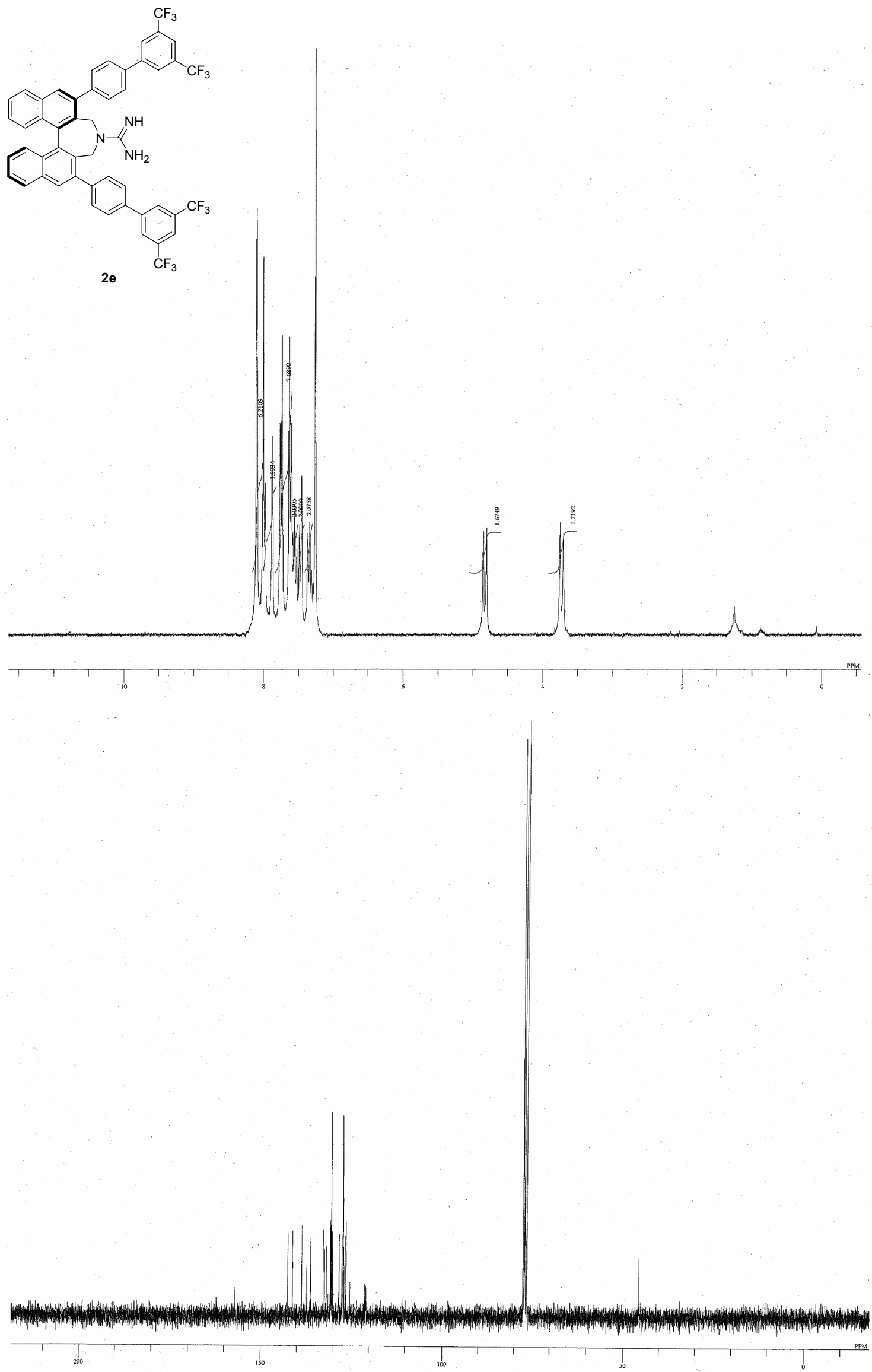

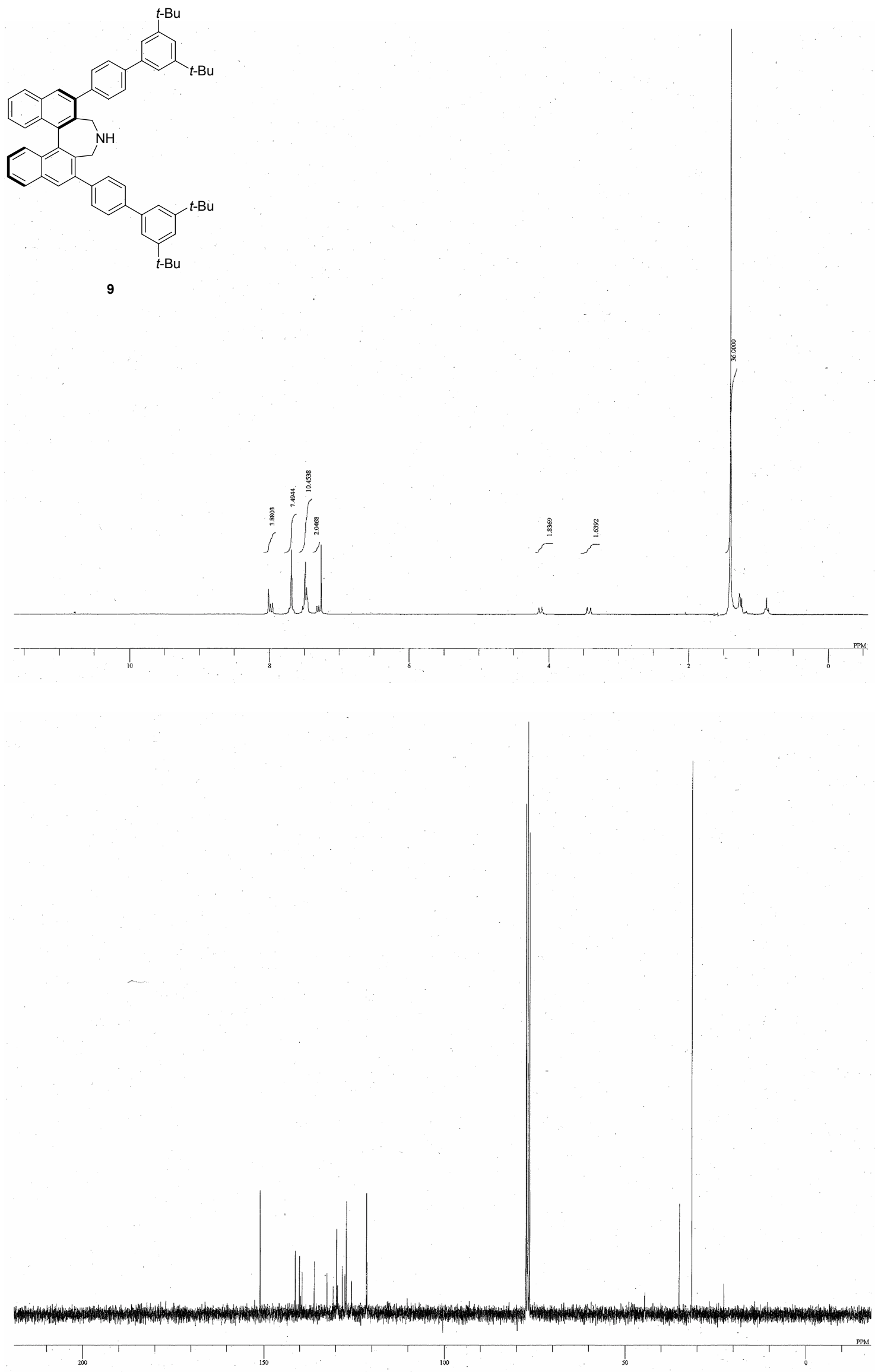


$$
\underbrace{\mathrm{CO}_{2} \mathrm{Et}}_{\mathbf{5 a}}
$$
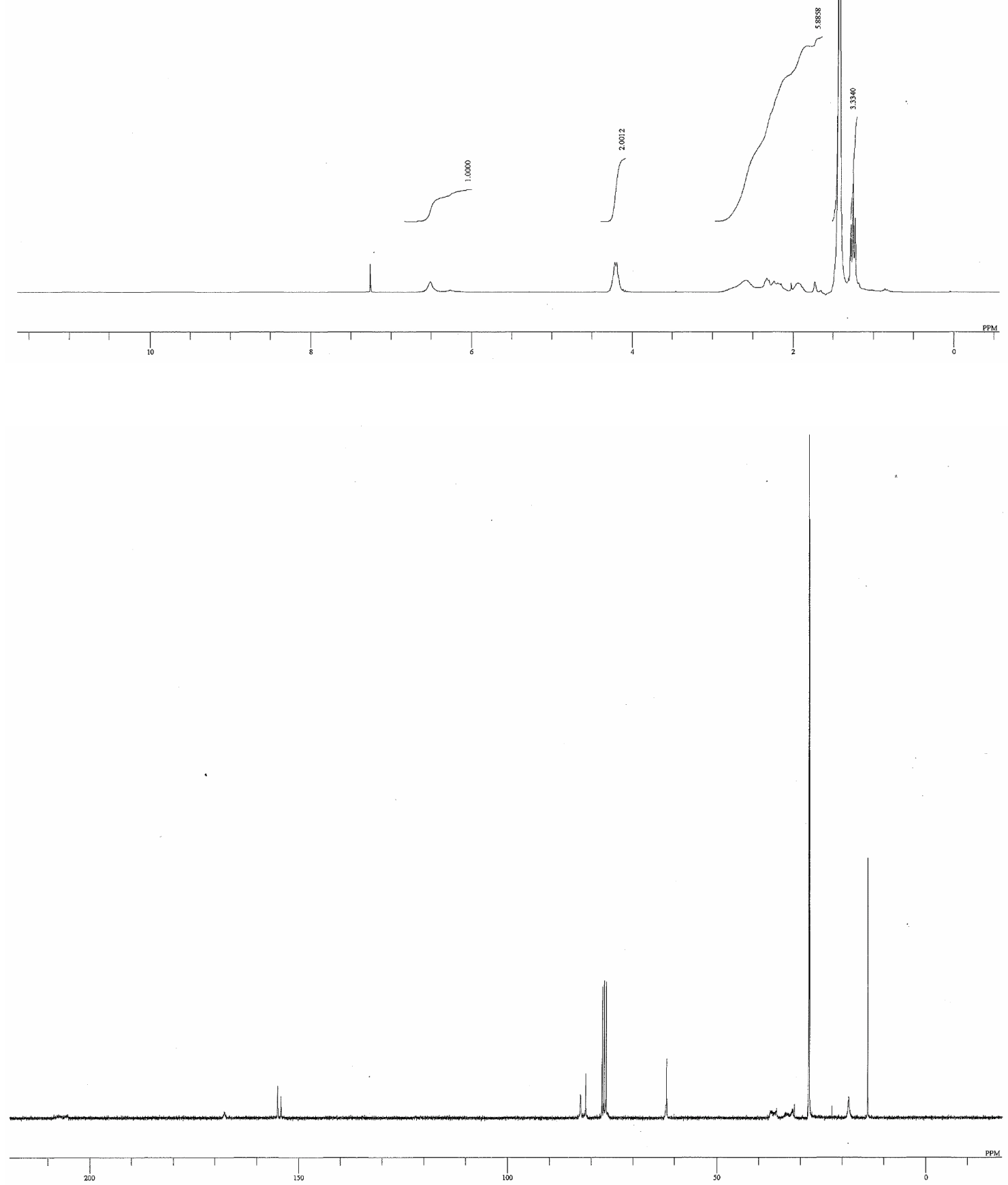

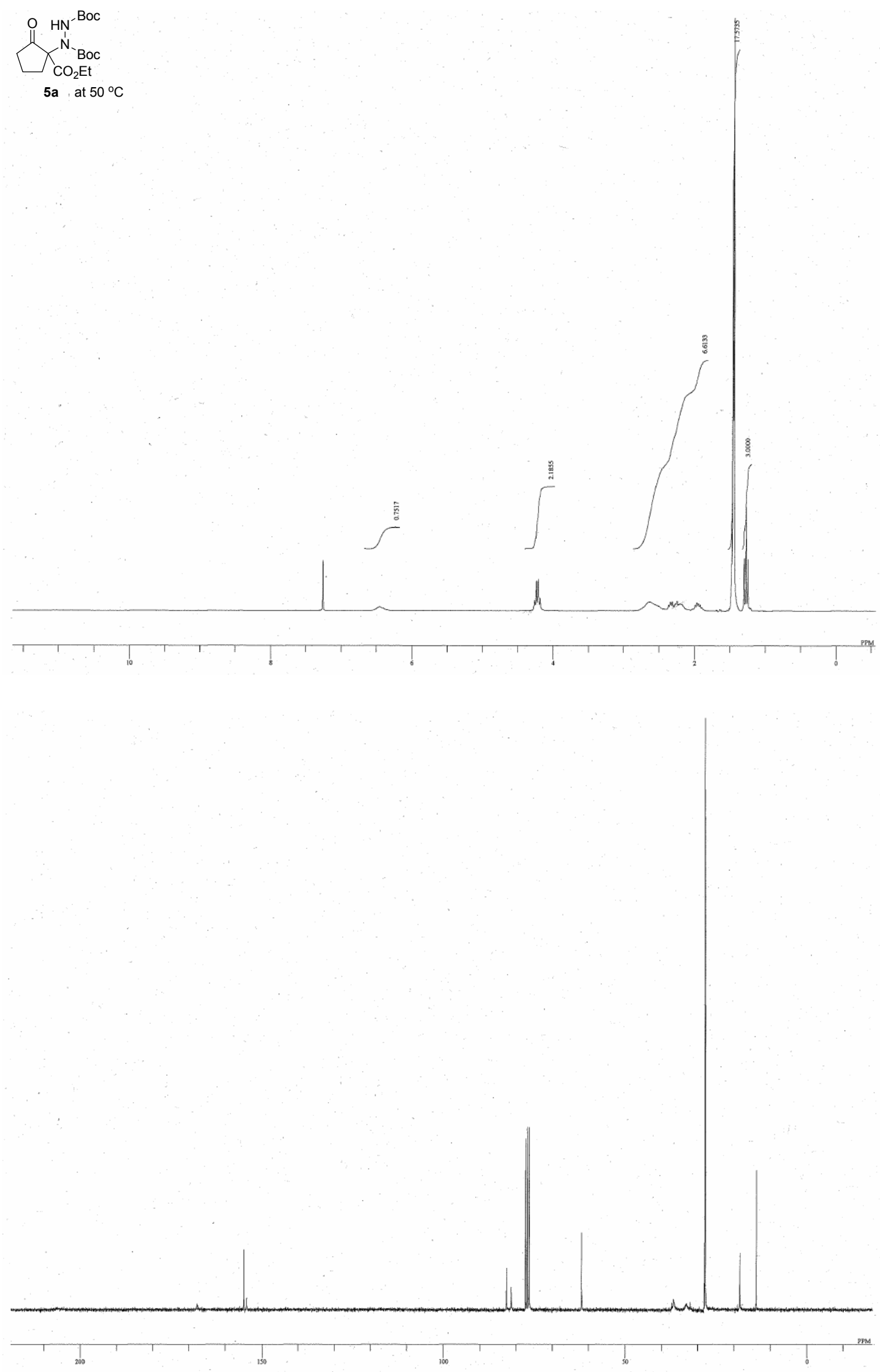

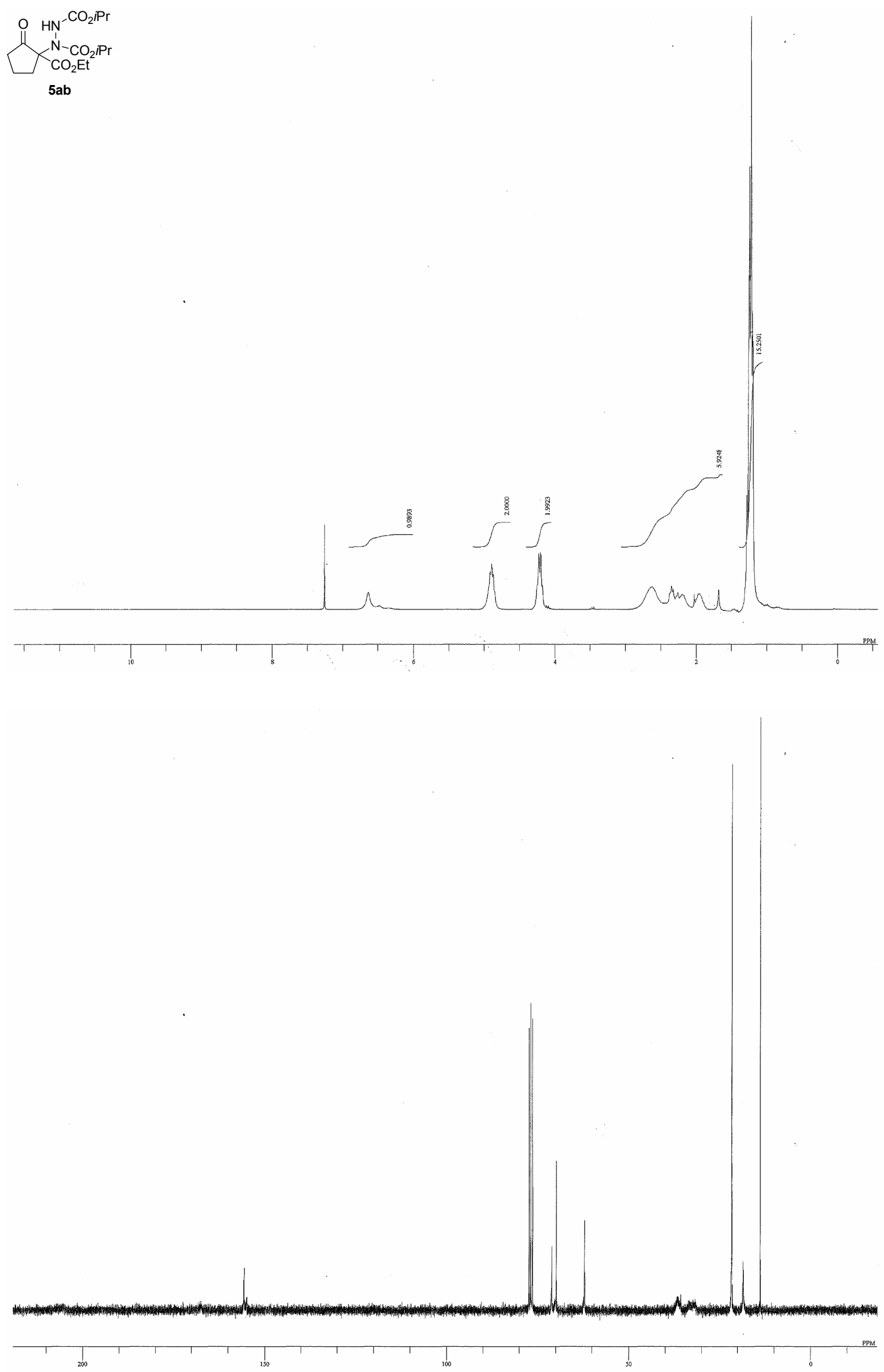


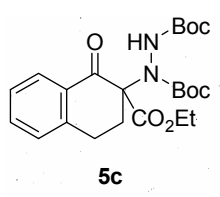

5c

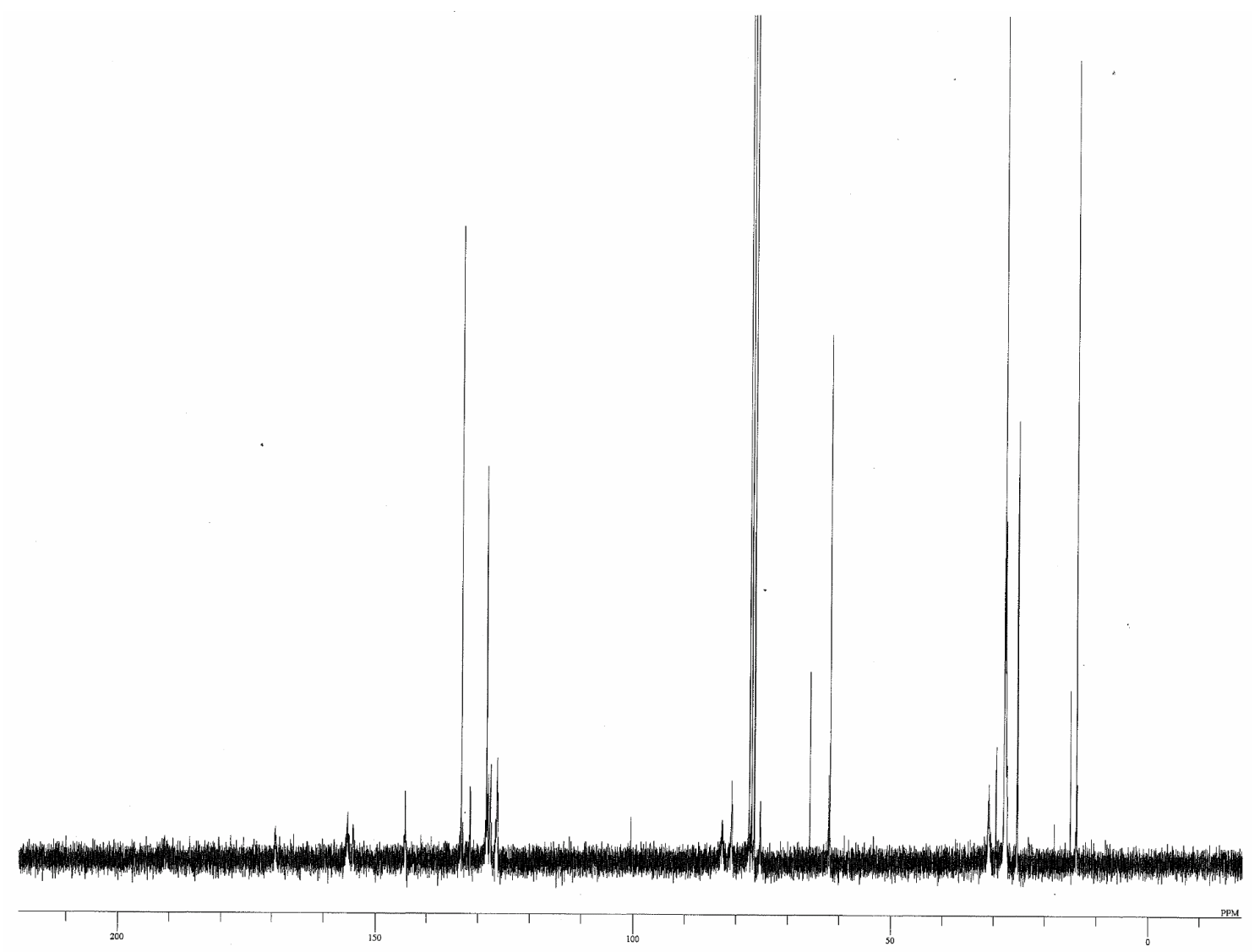




$$
\overbrace{\mathbf{5 d}}^{\mathrm{CO}_{2} \mathrm{Boc}^{\mathrm{N}}}
$$
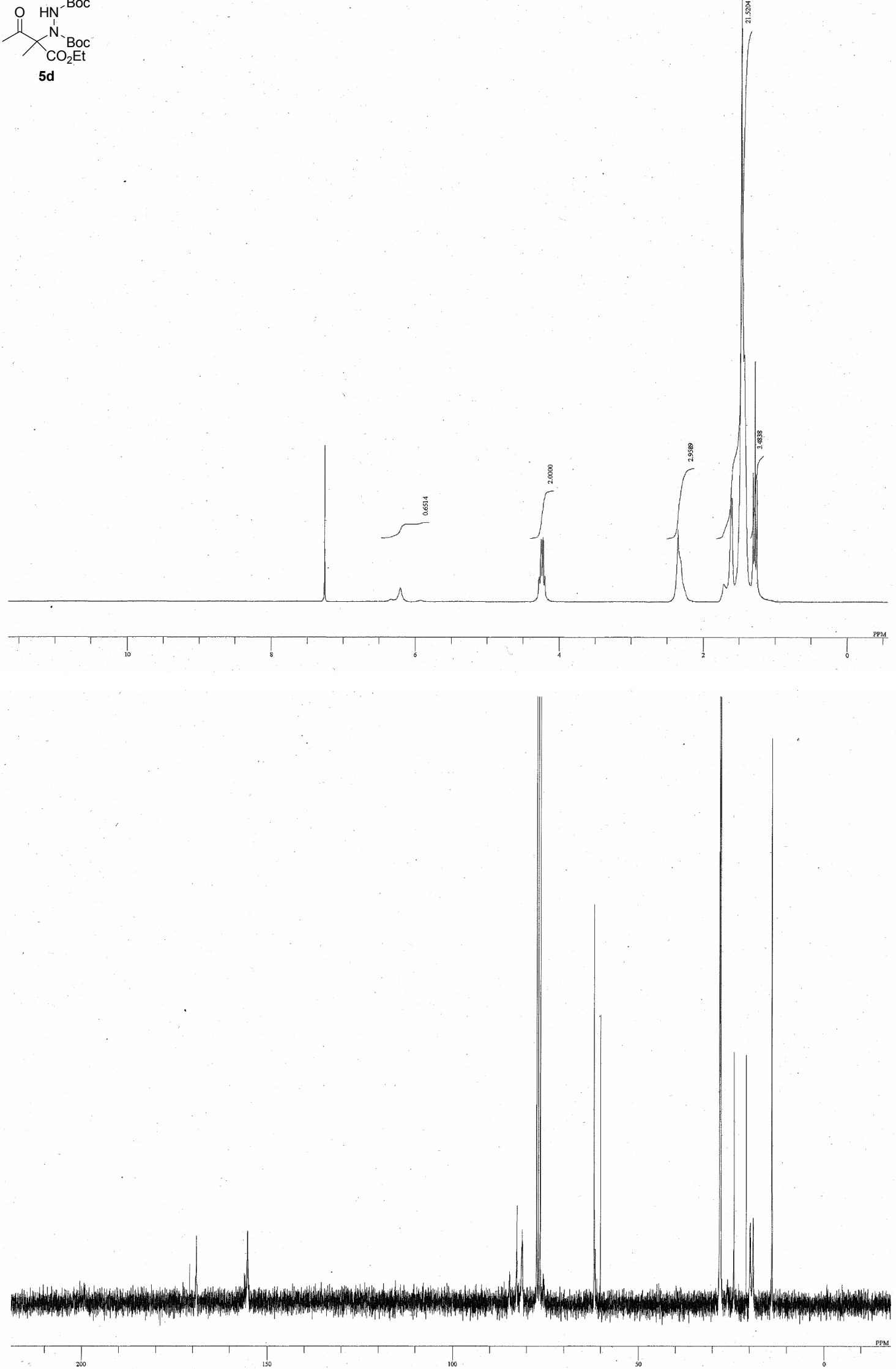


$$
\overbrace{5 e}^{\mathrm{CO}_{2} t-\mathrm{Bu}}
$$
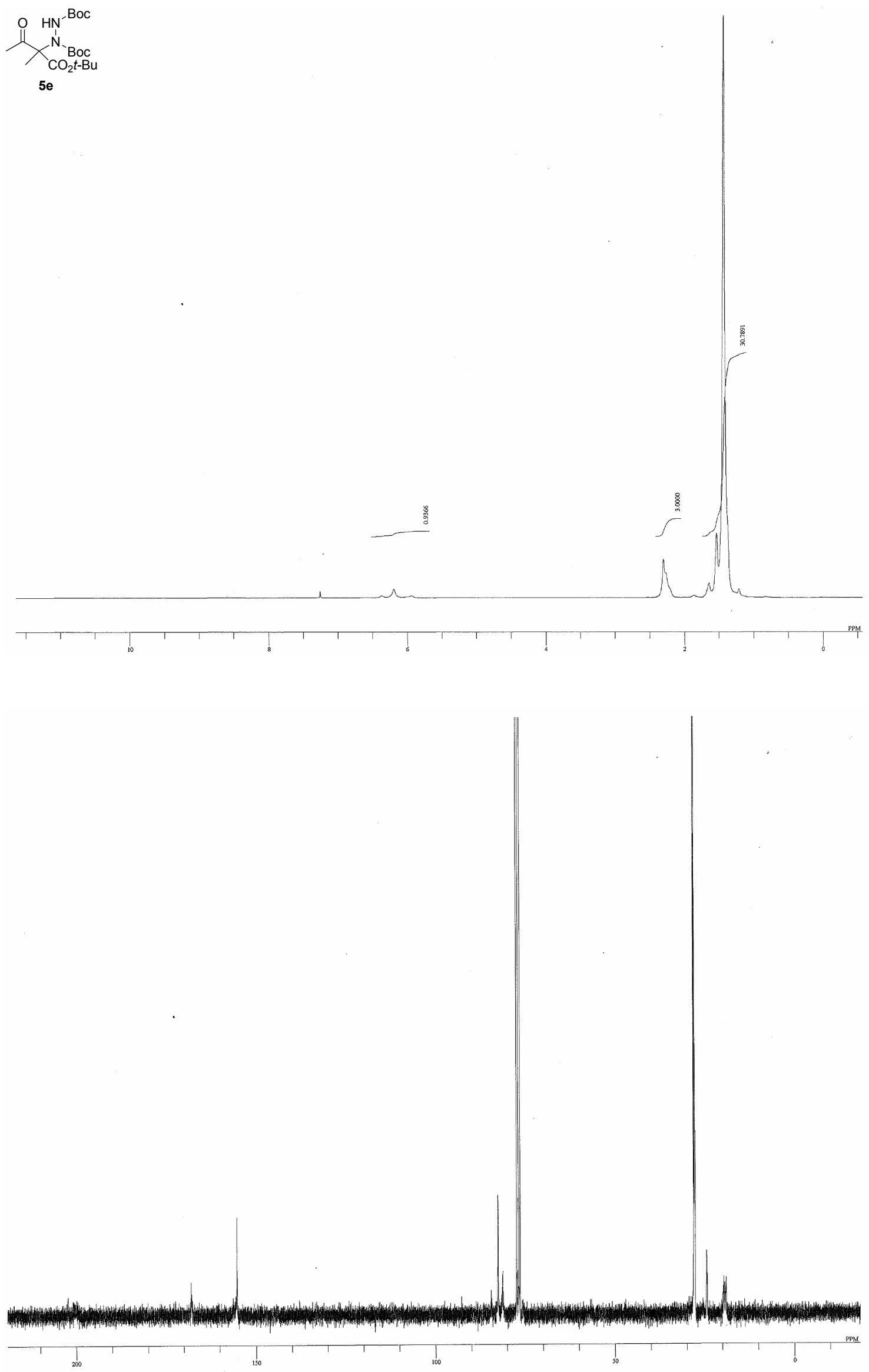


$$
\begin{aligned}
& \text { O } \mathrm{HN}^{-\mathrm{BOC}} \\
& \chi_{\mathrm{CO}_{2} t-\mathrm{Bu}}^{\mathrm{N}} \\
& \text { 5e at } 50^{\circ} \mathrm{C}
\end{aligned}
$$
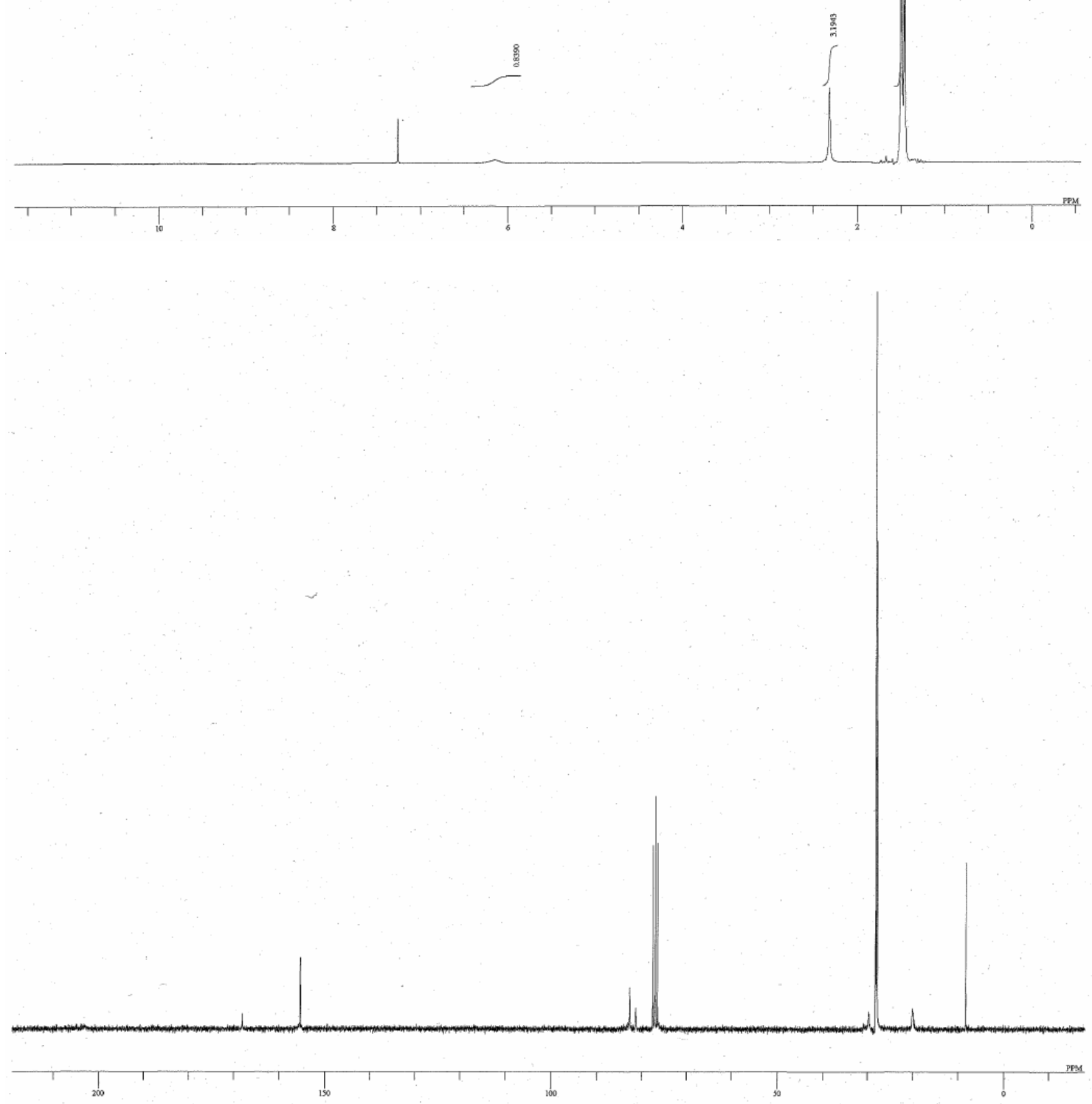


$$
\overbrace{\mathbf{5 f}}^{O} \underbrace{\mathrm{N}}_{\mathrm{CO}_{2} t-\mathrm{Bu}}
$$

1
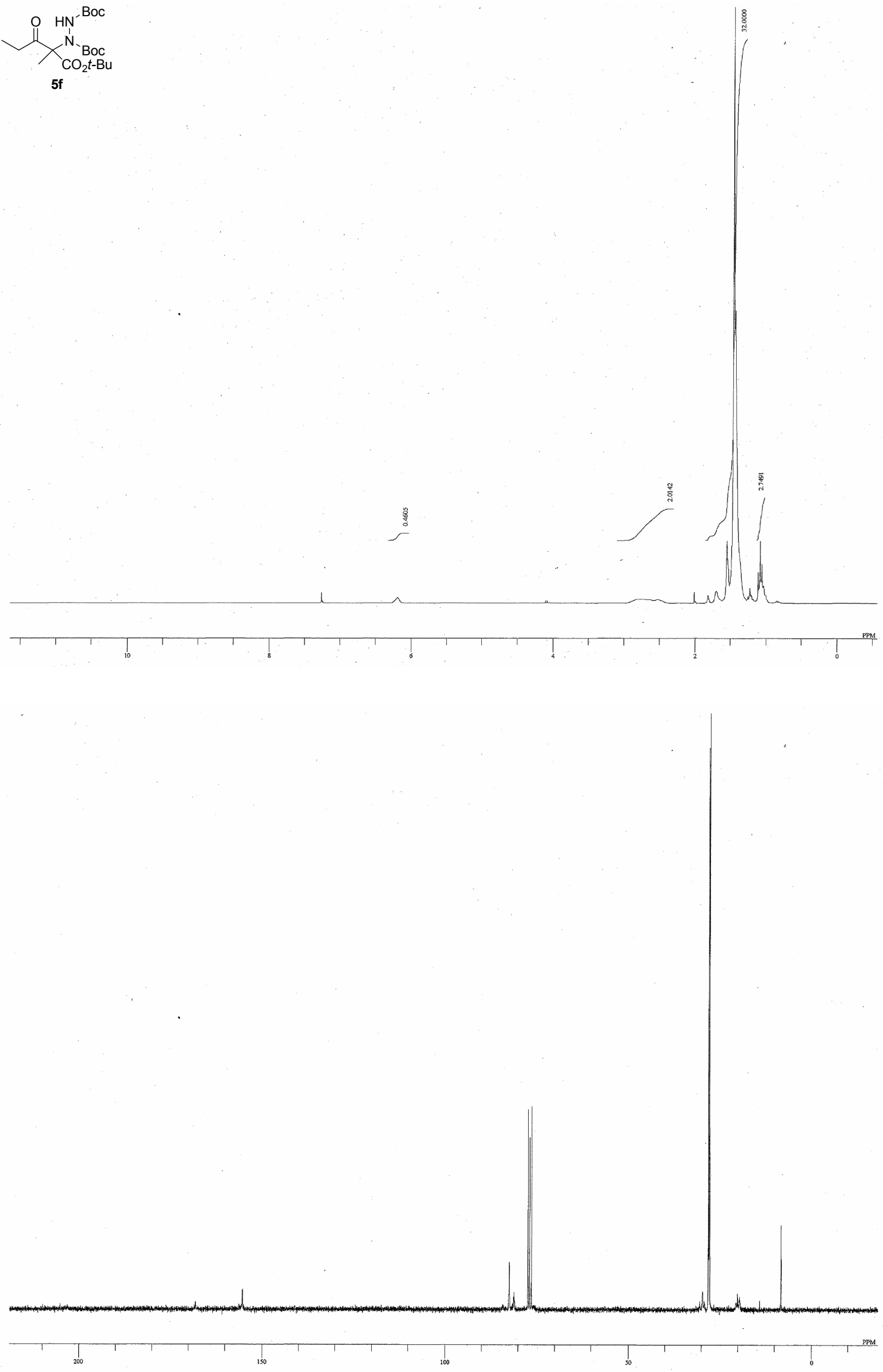


$$
\begin{aligned}
& \mathrm{O}^{\mathrm{HN}^{-\mathrm{BOC}}} \\
& \mathrm{CO}_{2} t-\mathrm{Bu} \\
& 5 f \text { at } 50^{\circ} \mathrm{C}
\end{aligned}
$$

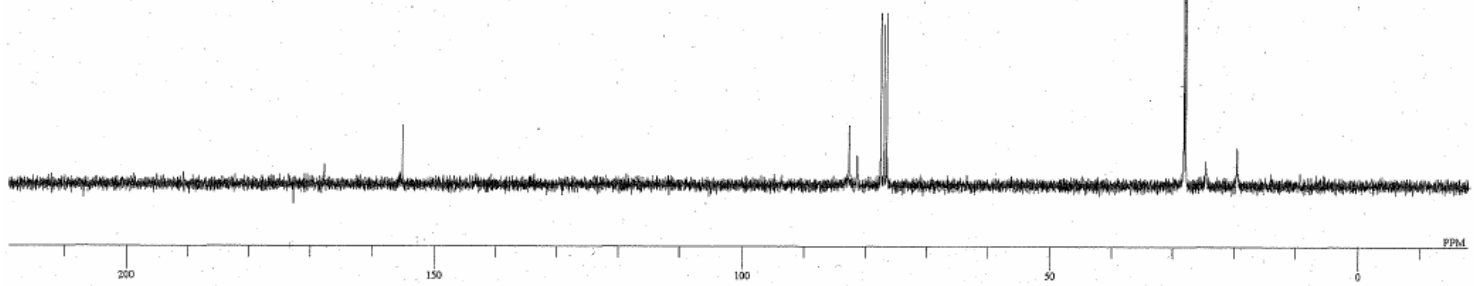




$$
\overbrace{\mathbf{E t}}^{\mathrm{O}} \underbrace{\mathrm{N}}_{\mathrm{CO}_{2} t-\mathrm{Bu}} \mathrm{HN}^{-\mathrm{Boc}}
$$
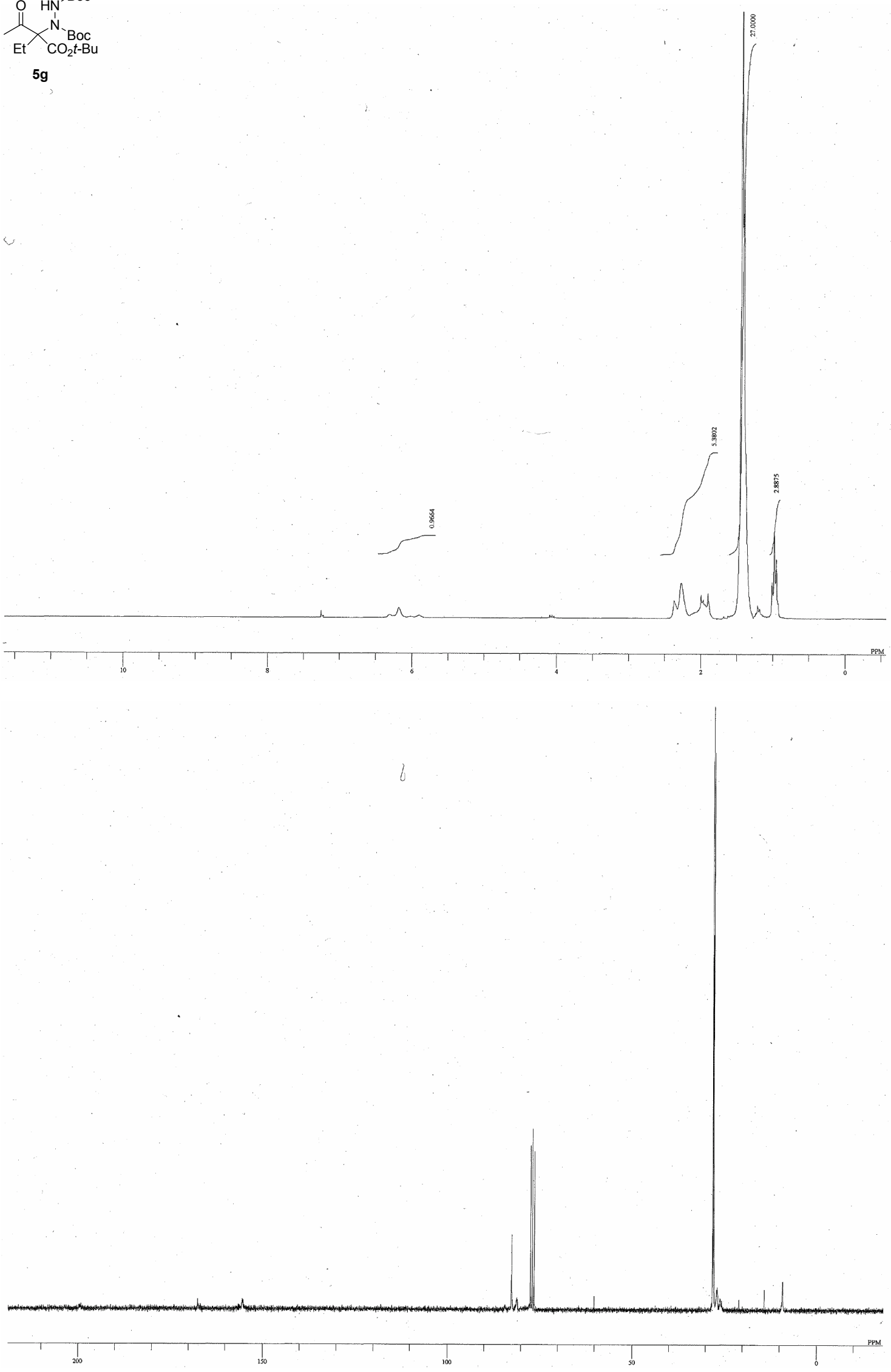


$$
\underbrace{\mathrm{CO}_{2} t-\mathrm{Bu}}_{\mathbf{5 h}}
$$
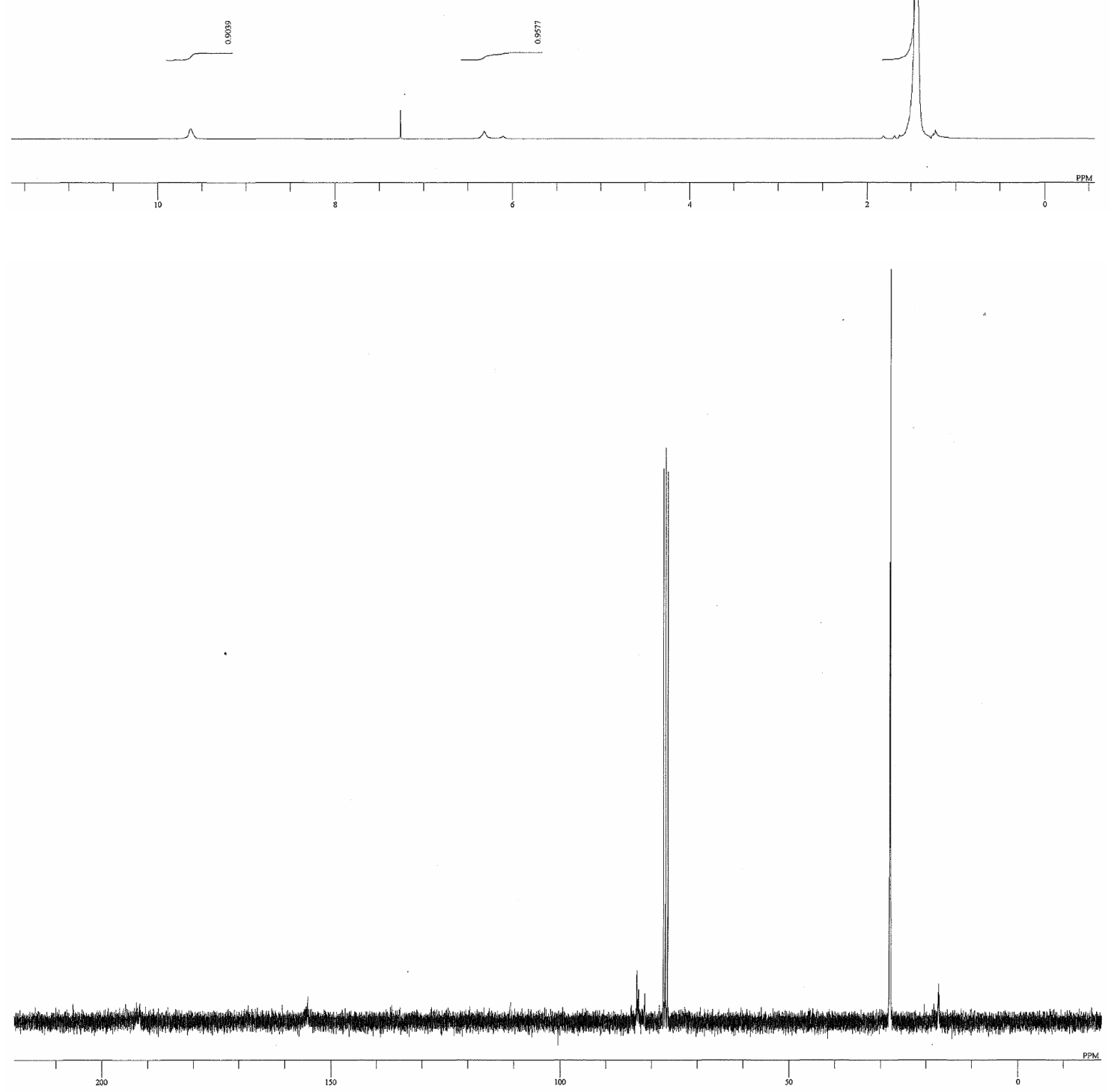

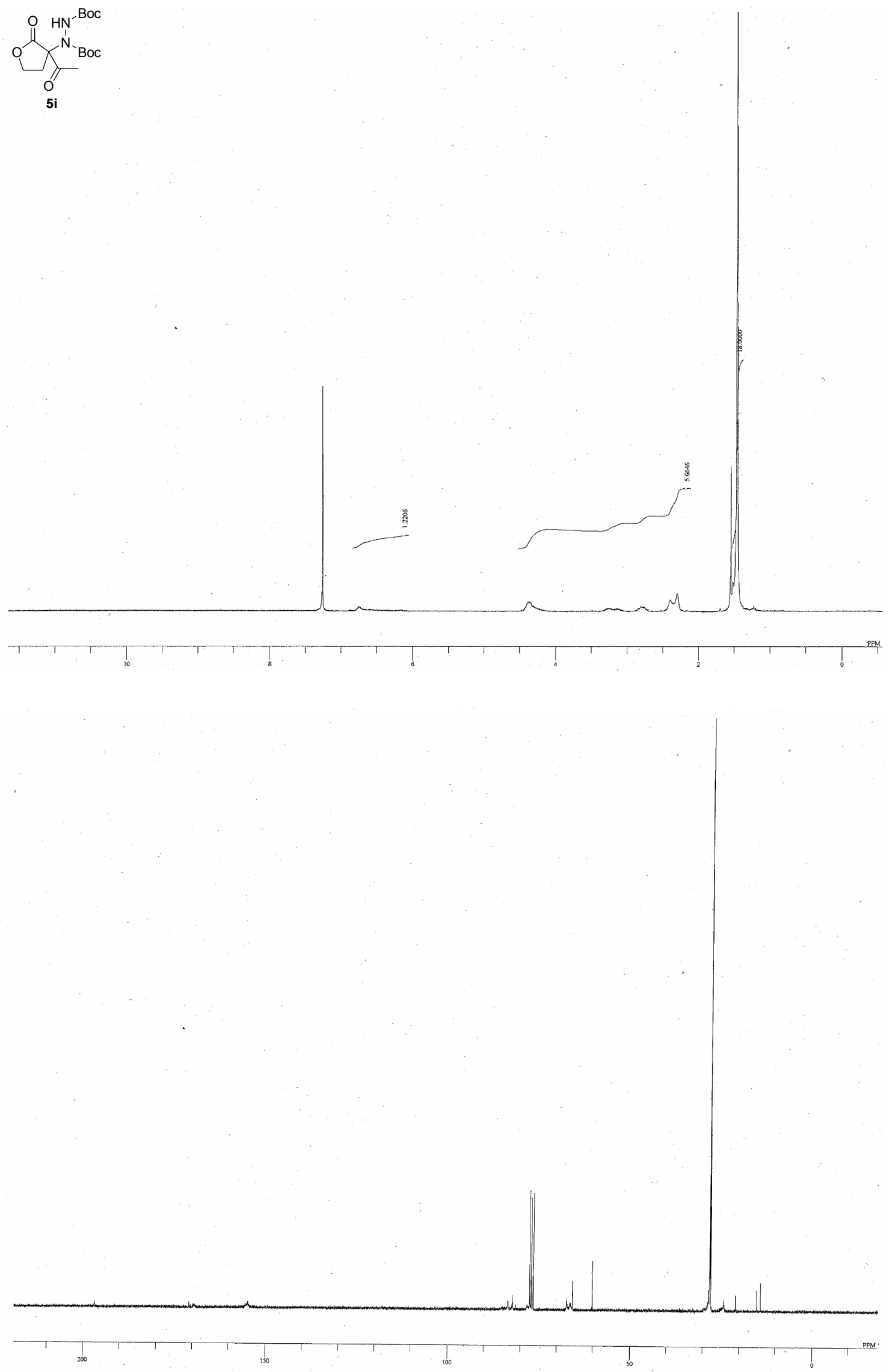

S33 

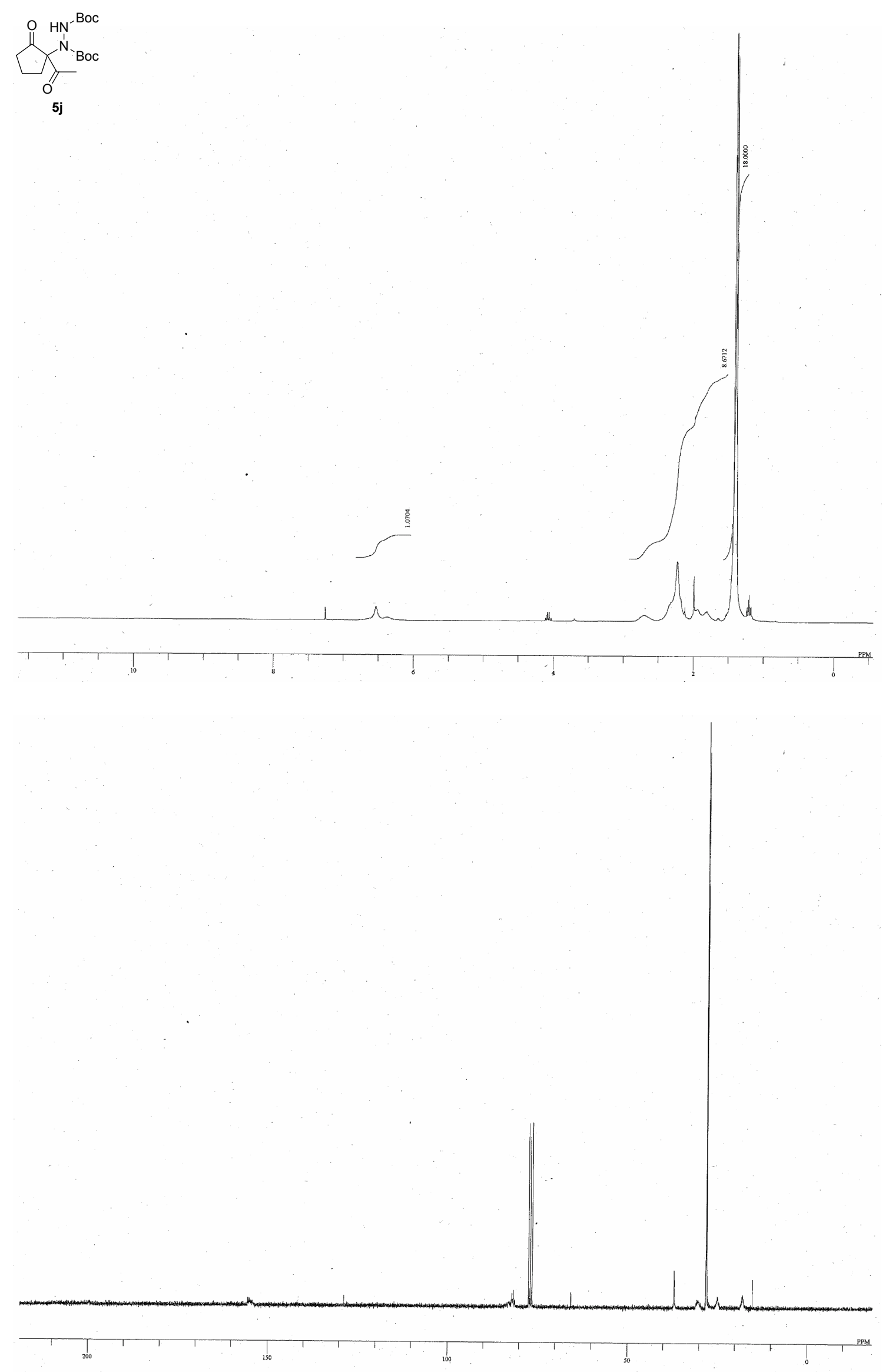


$$
\overbrace{10 \mathrm{a}}^{\mathrm{CO}_{2} \mathrm{Me}}
$$
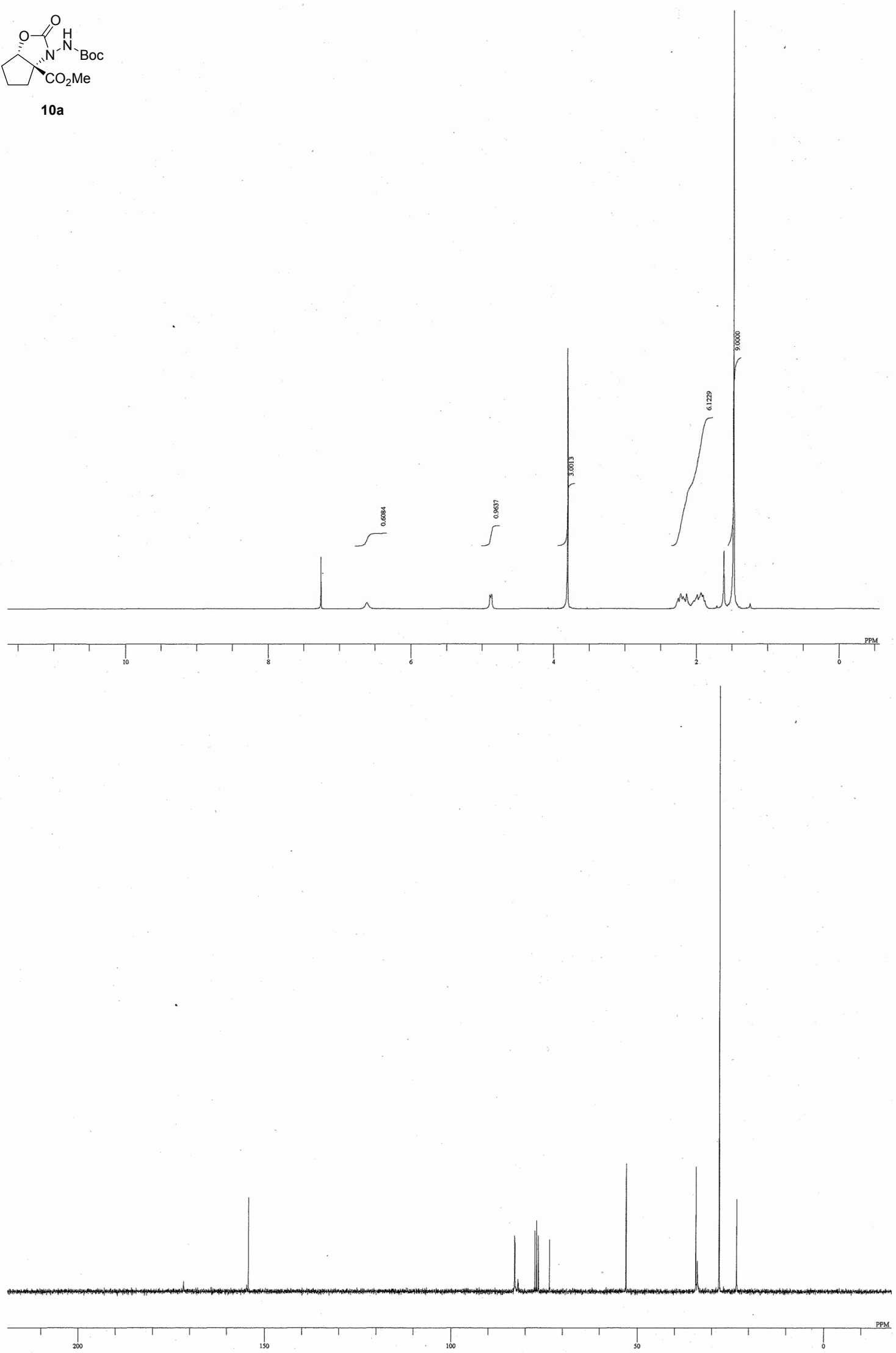

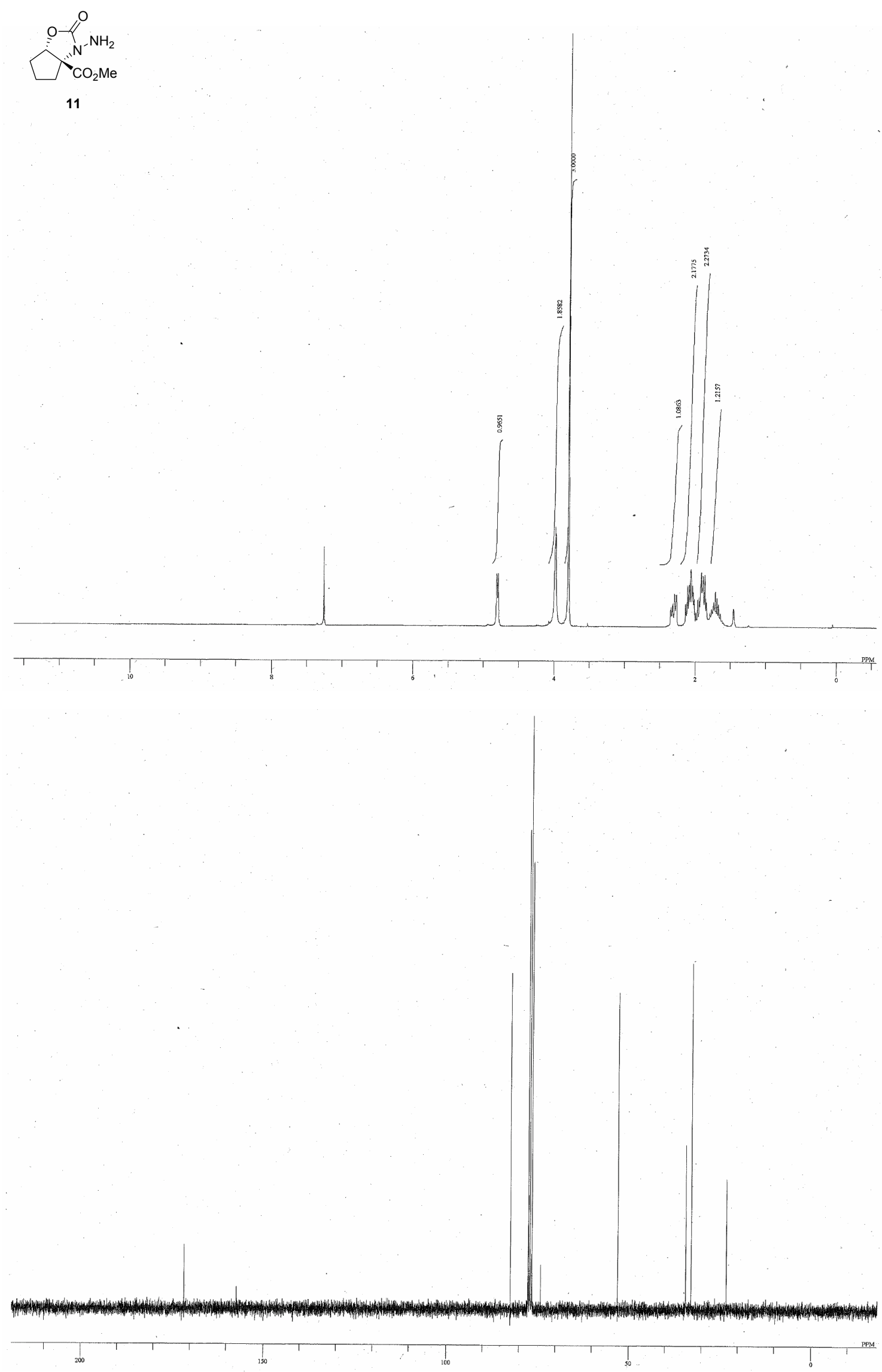

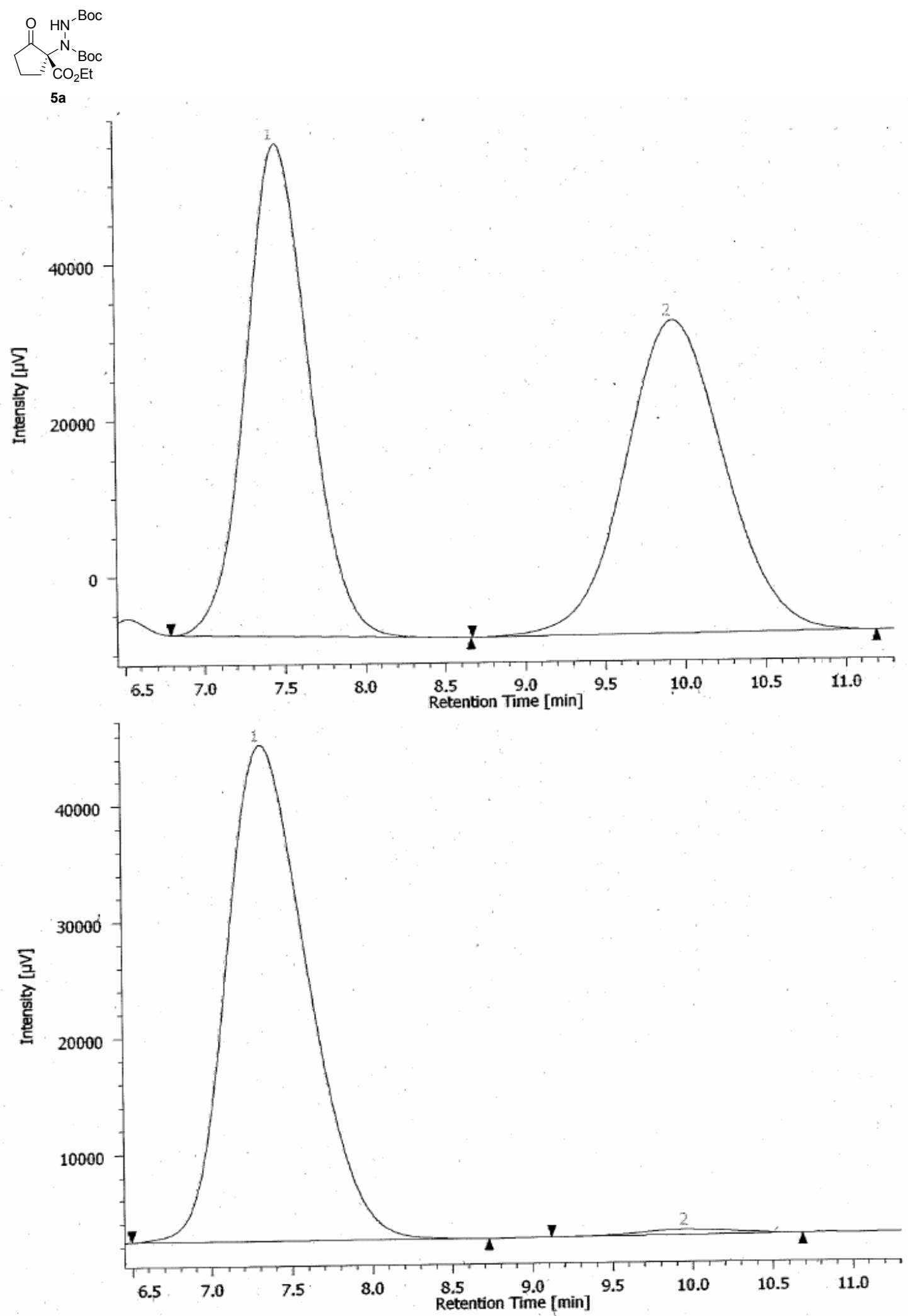

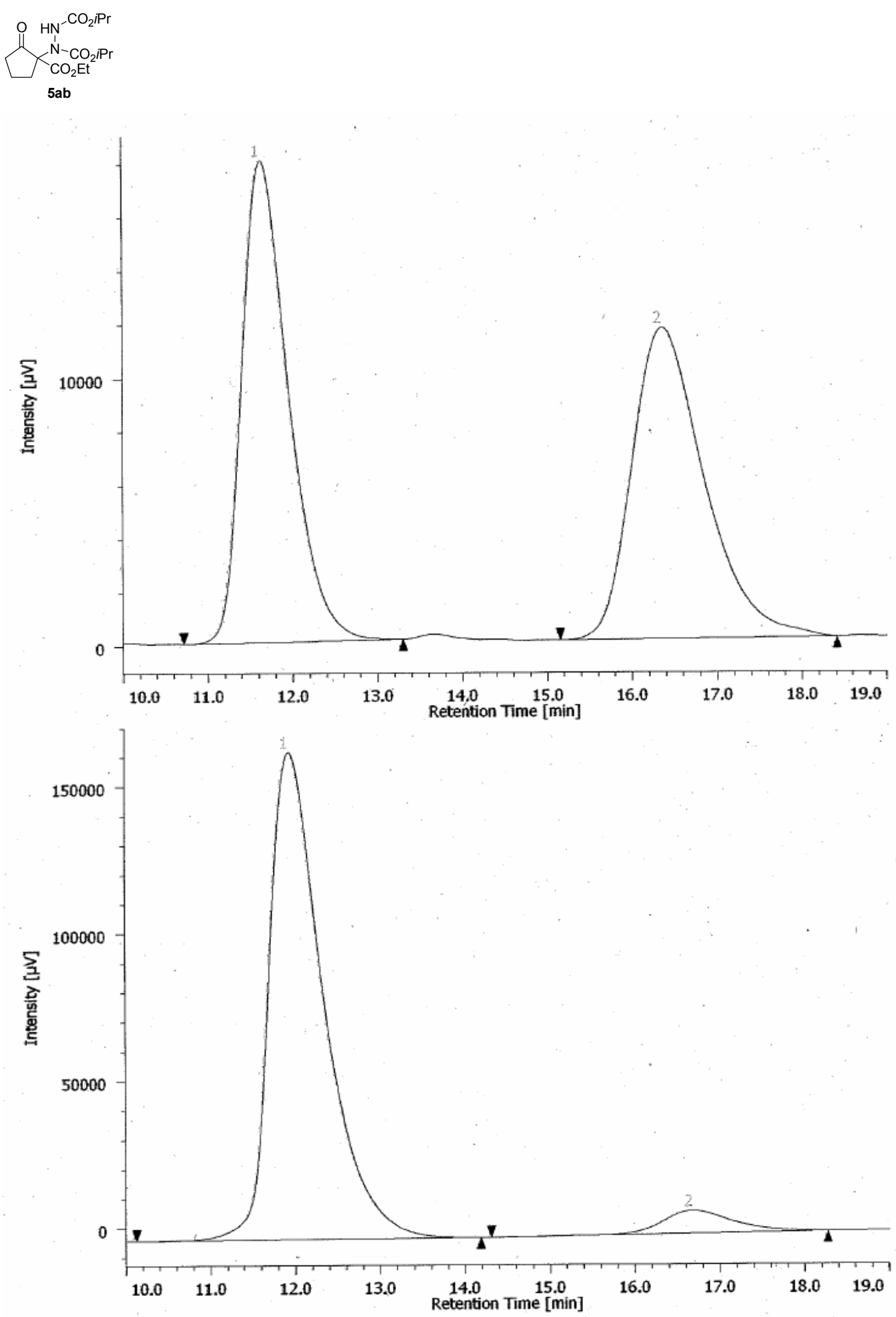
$\underbrace{1}_{\mathbf{5 a c}}$
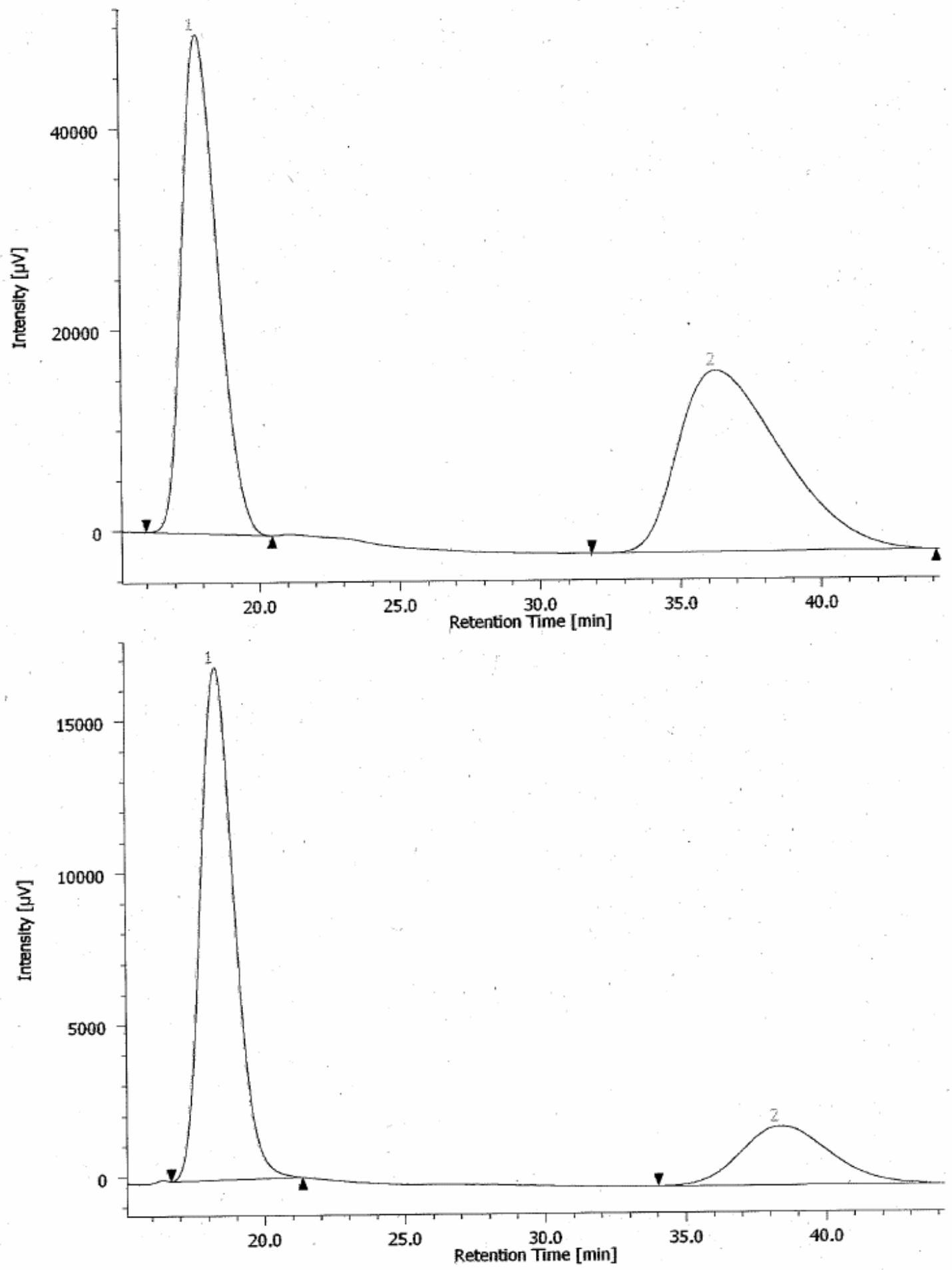
$\overbrace{\mathrm{CO}_{2} \mathrm{Et}}^{\mathrm{N}}$

$5 b$
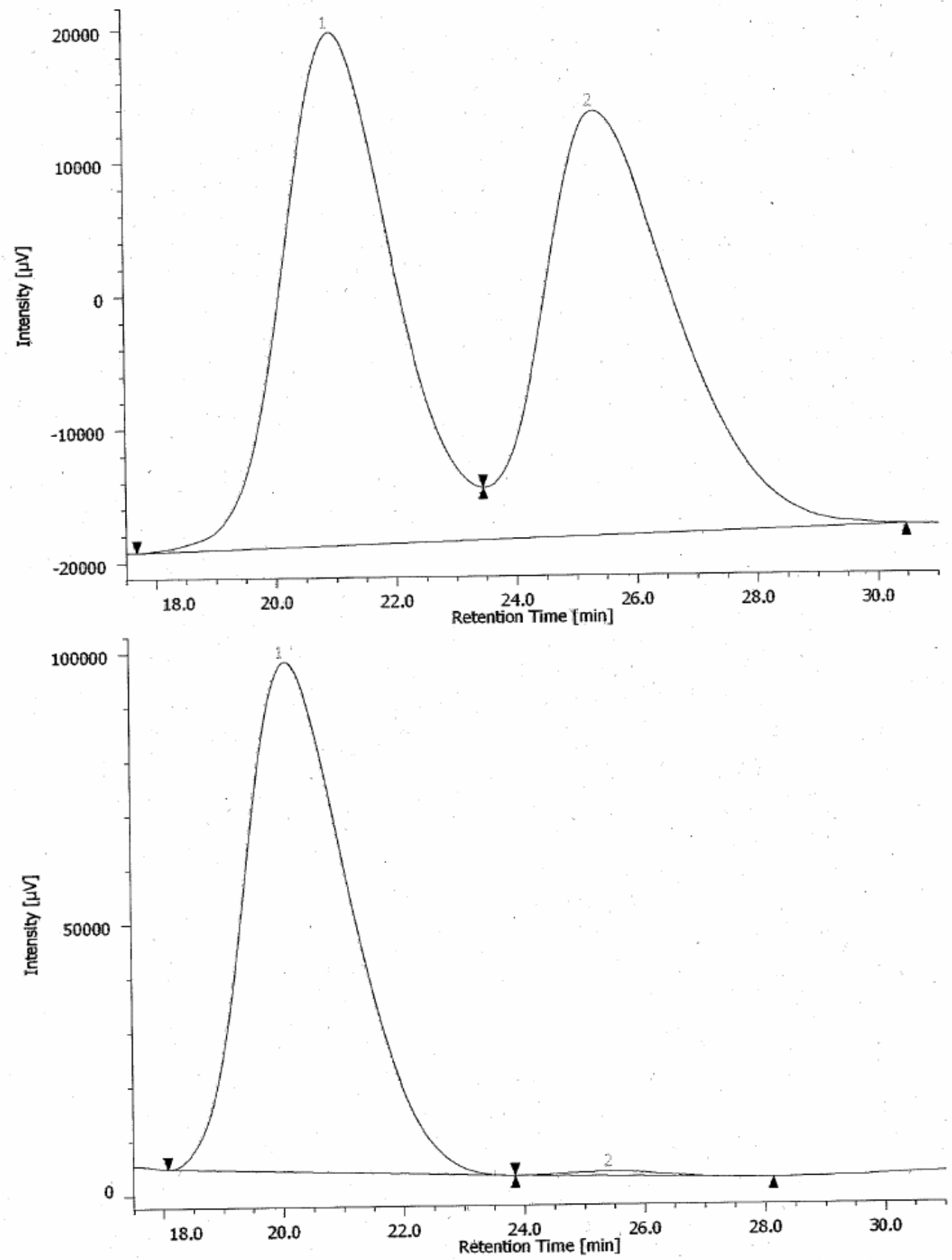
<smiles>CC1CC([N+](=O)[O-])C(=O)c2ccccc21</smiles>
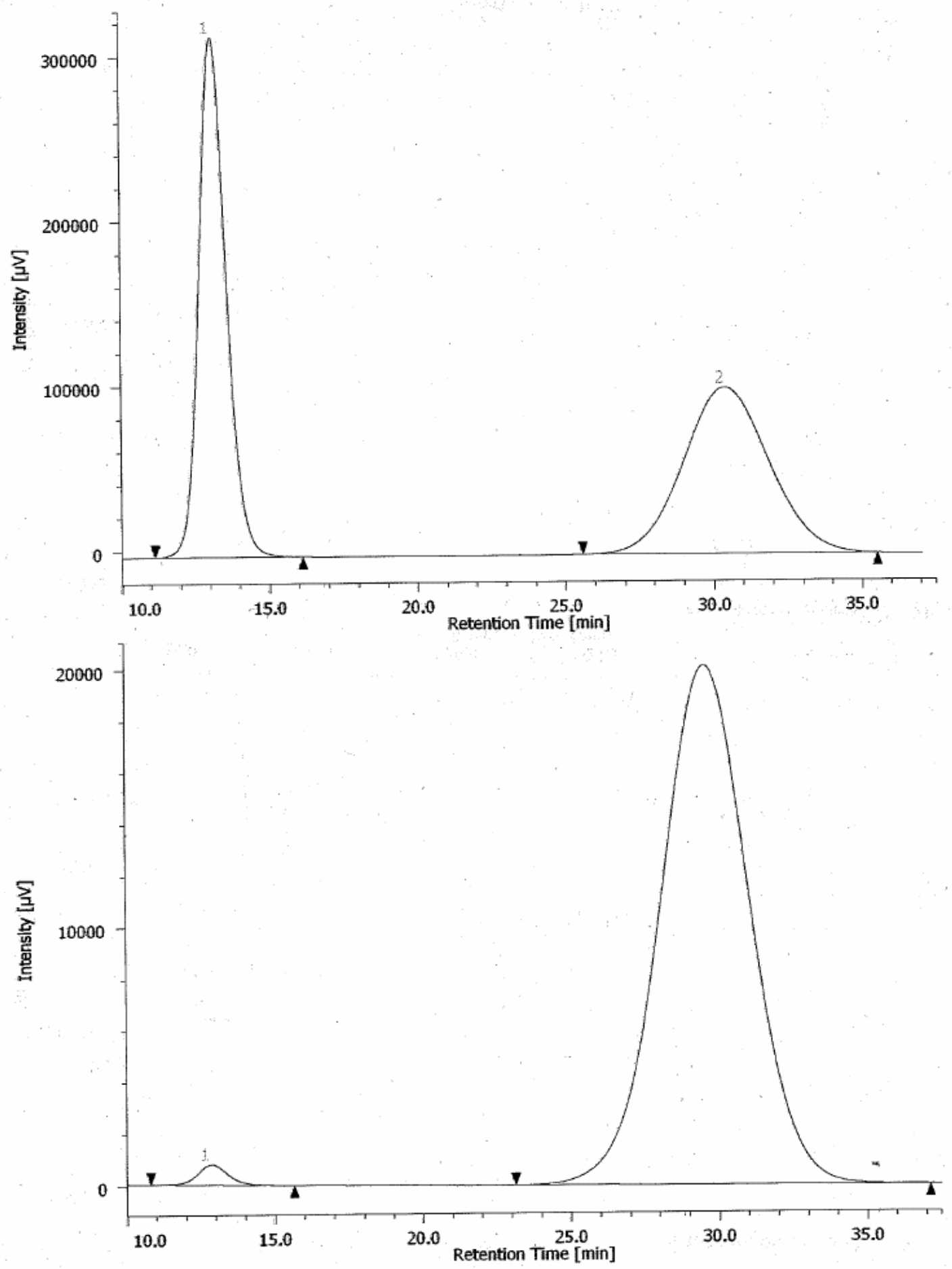
$\underbrace{\mathrm{N}}_{\mathrm{CO}_{2} \mathrm{Et}}$

$5 d$
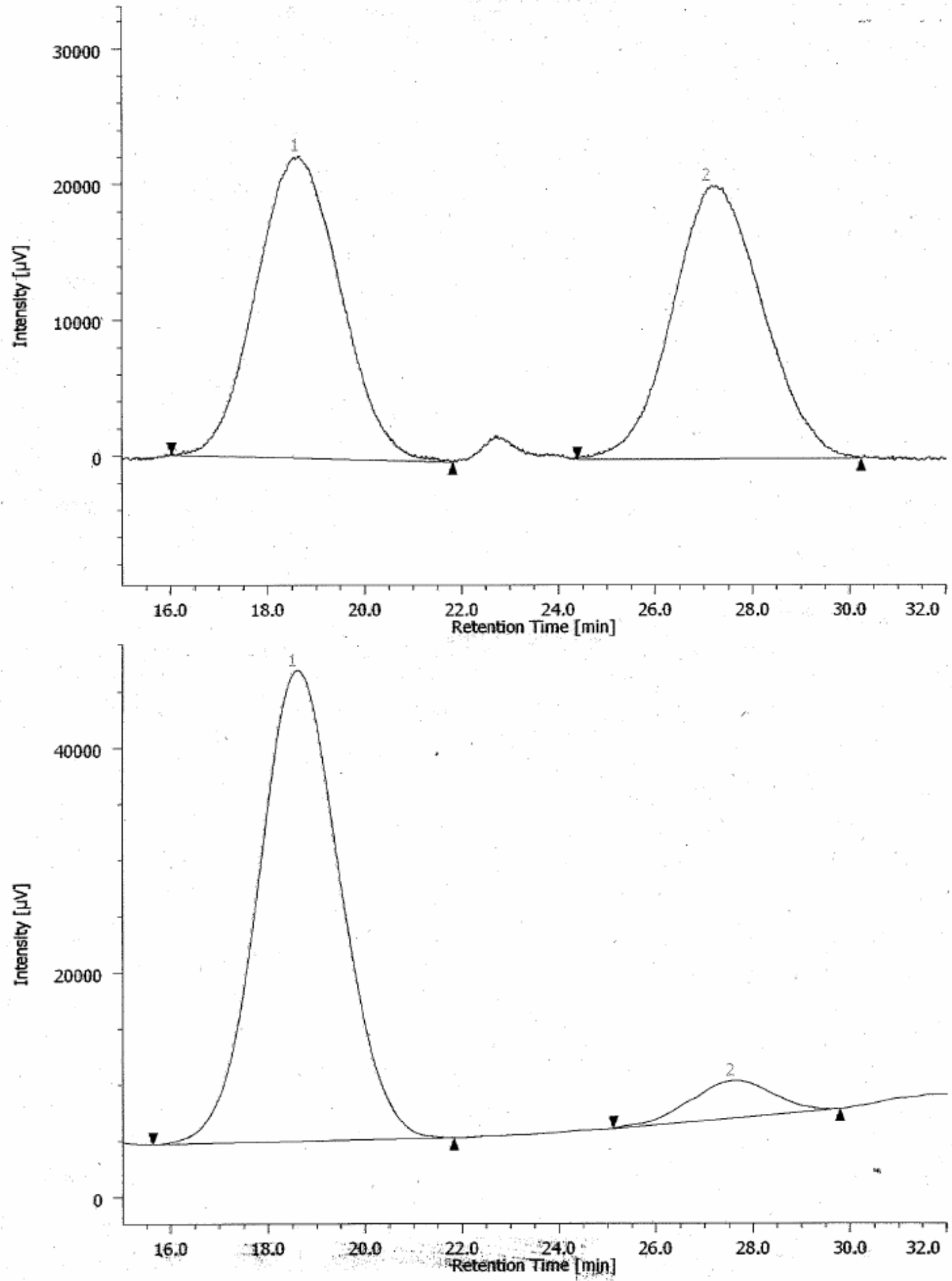
$\overbrace{\mathrm{CO}_{2} t-\mathrm{Bu}}^{\mathrm{N}}{ }_{-\mathrm{Boc}}^{\mathrm{H}}$

$5 e$
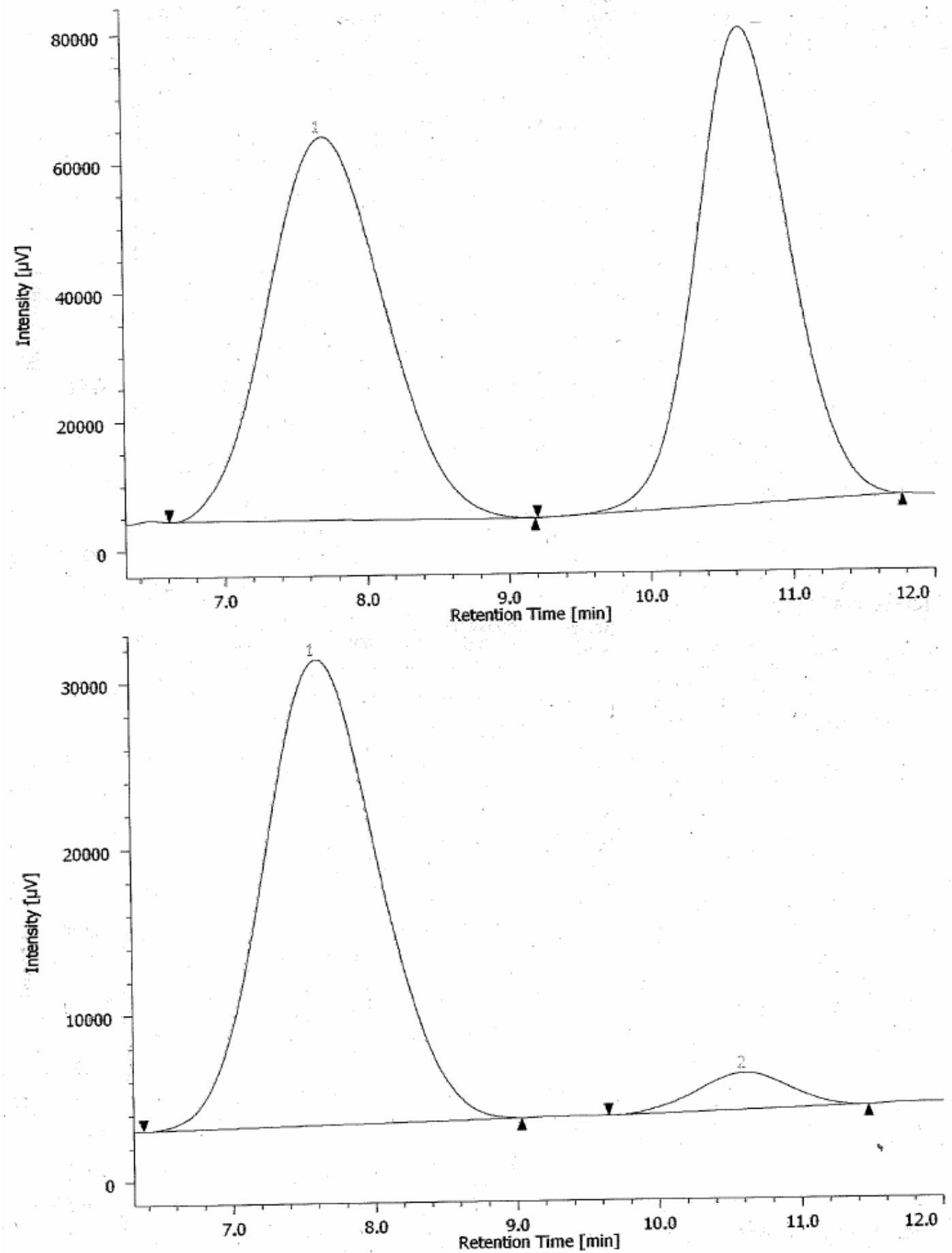
$\overbrace{\mathbf{5}}^{\mathrm{CO}_{2} t-\mathrm{Bu}}$
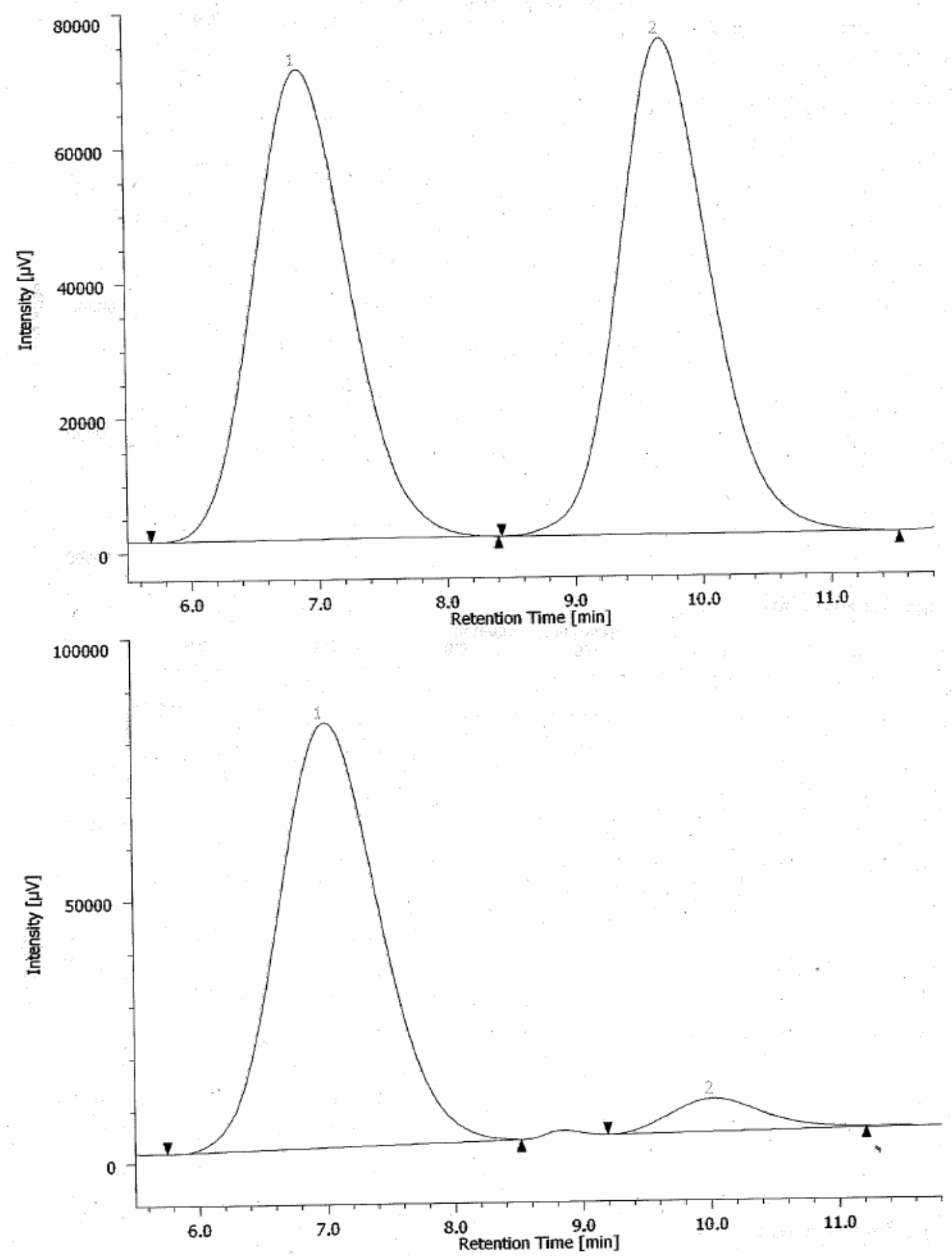
$\underbrace{\mathrm{O}}_{\mathrm{Et}} \mathrm{CO}_{\mathrm{CO}_{2} t-\mathrm{Bu}}^{\mathrm{N}} \mathrm{HN}_{-}^{-\mathrm{Boc}}$

$5 \mathrm{~g}$
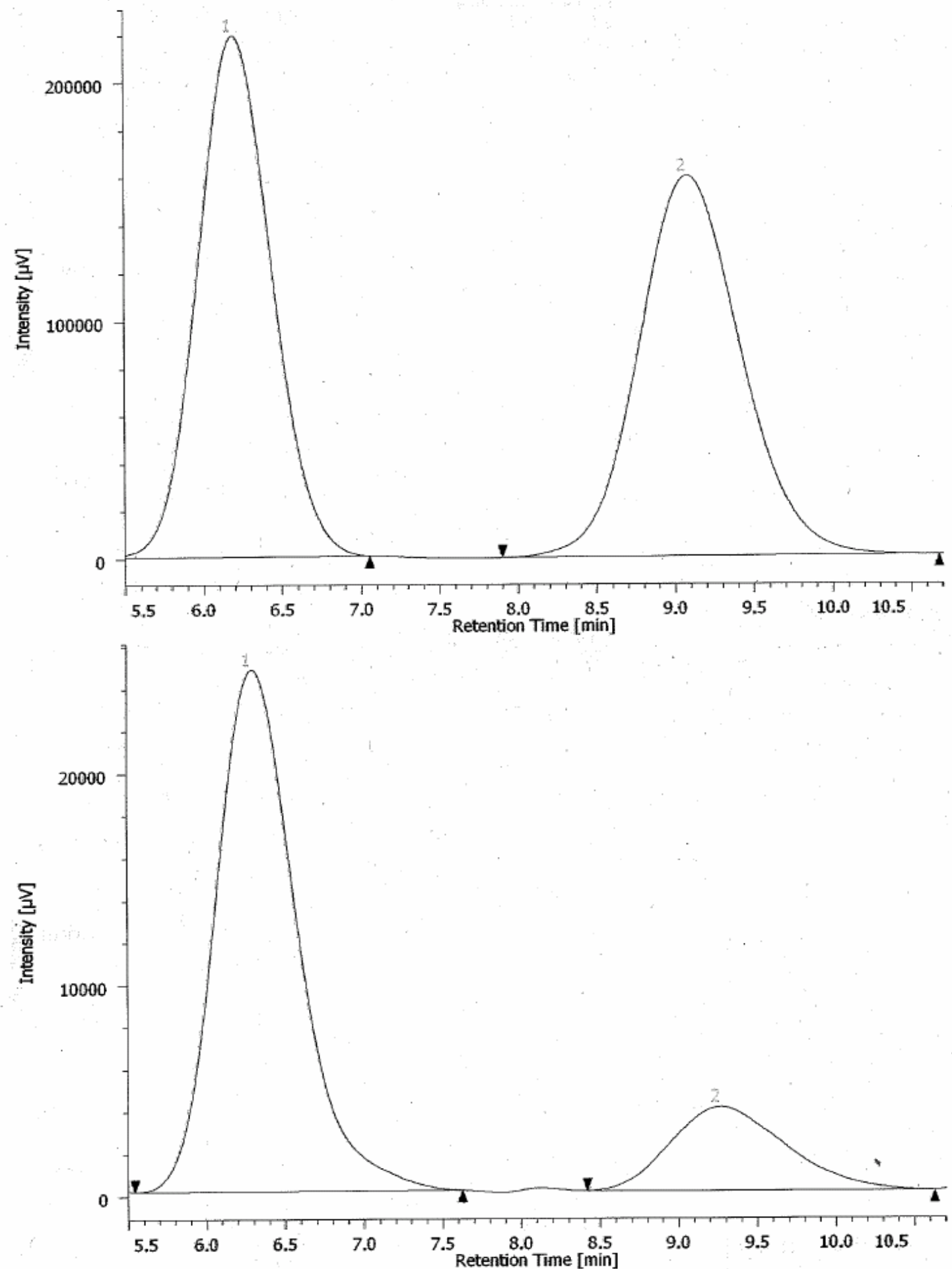

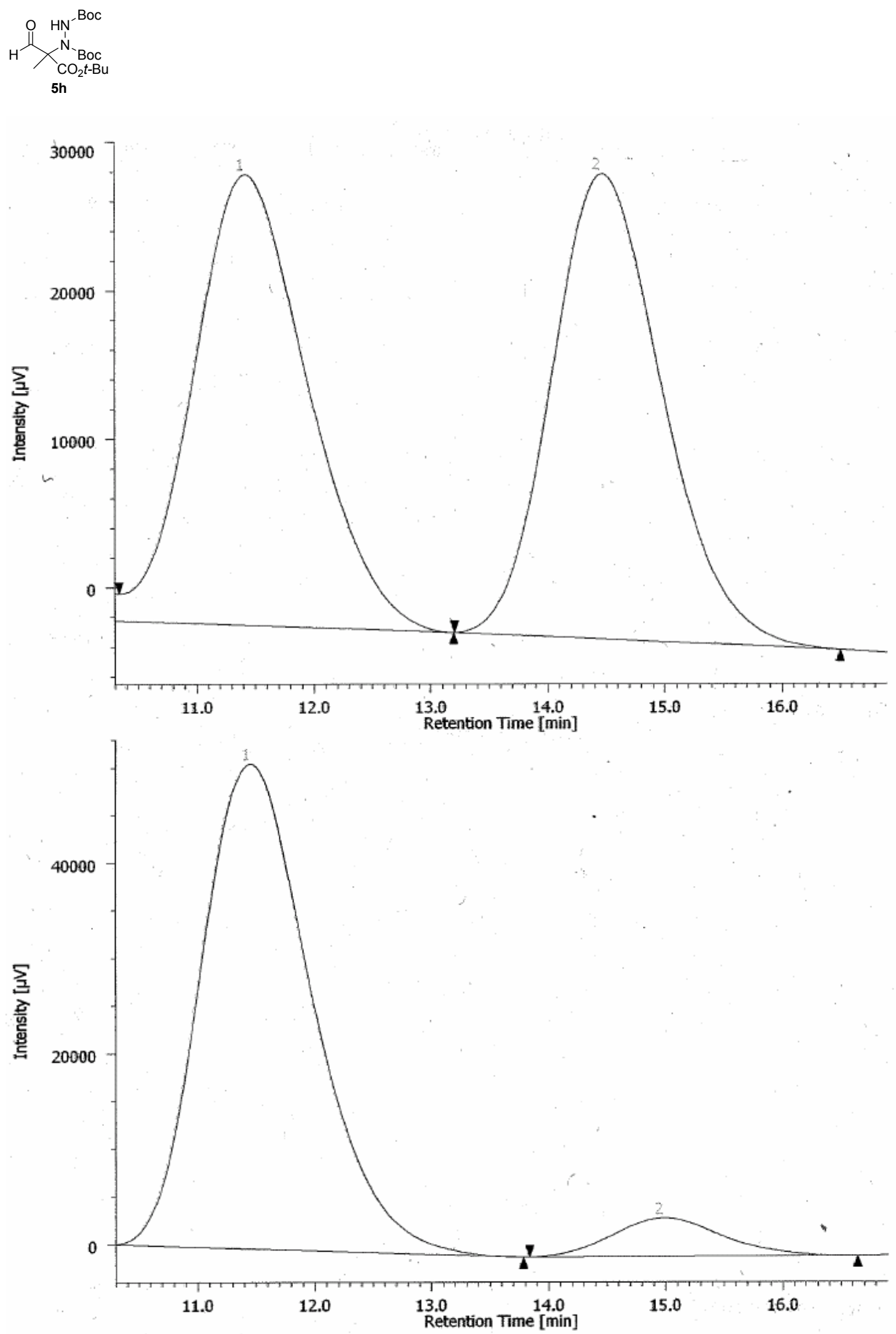
$\underbrace{N_{i}^{N}}_{\mathbf{5 i}}$
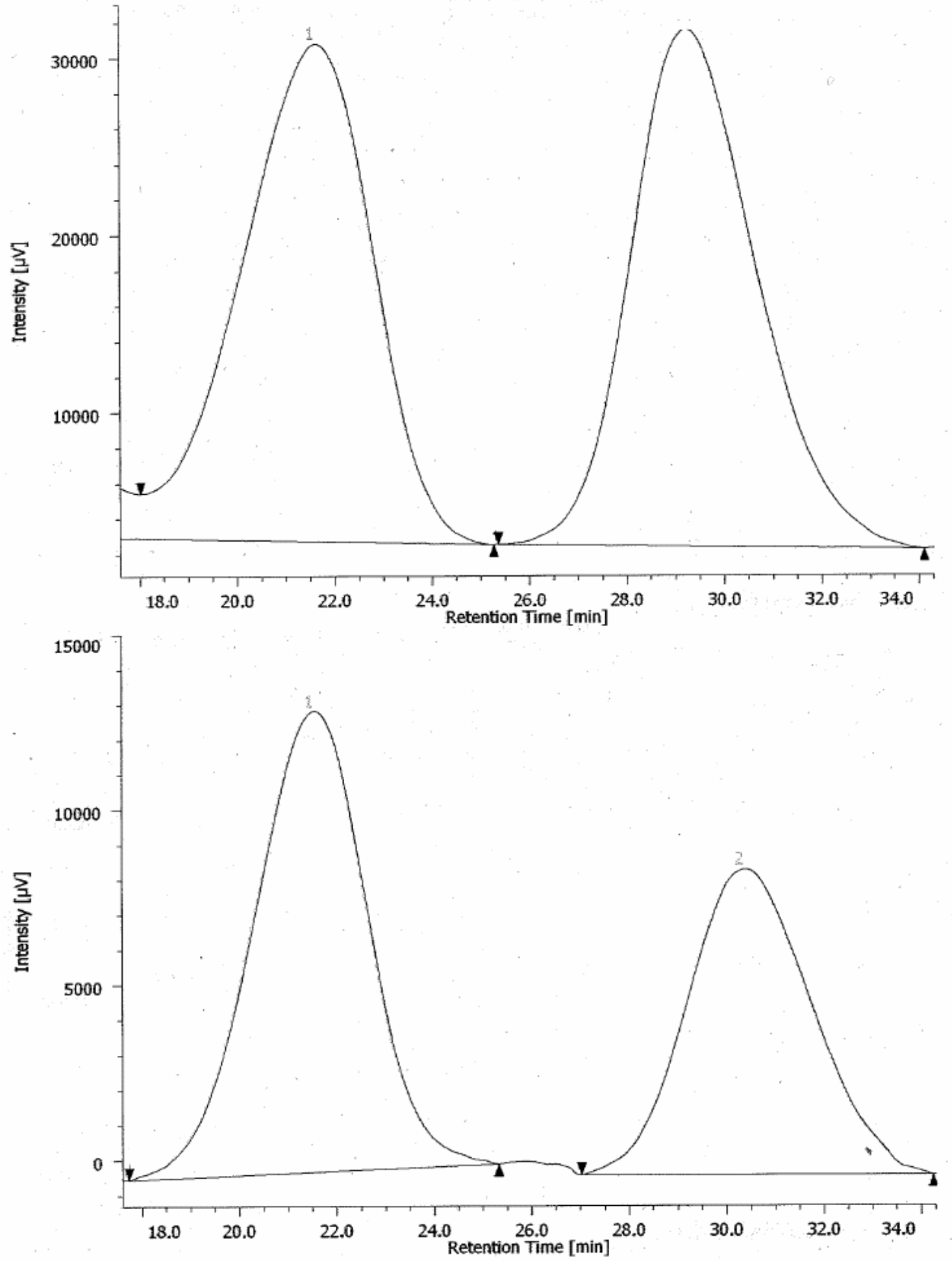

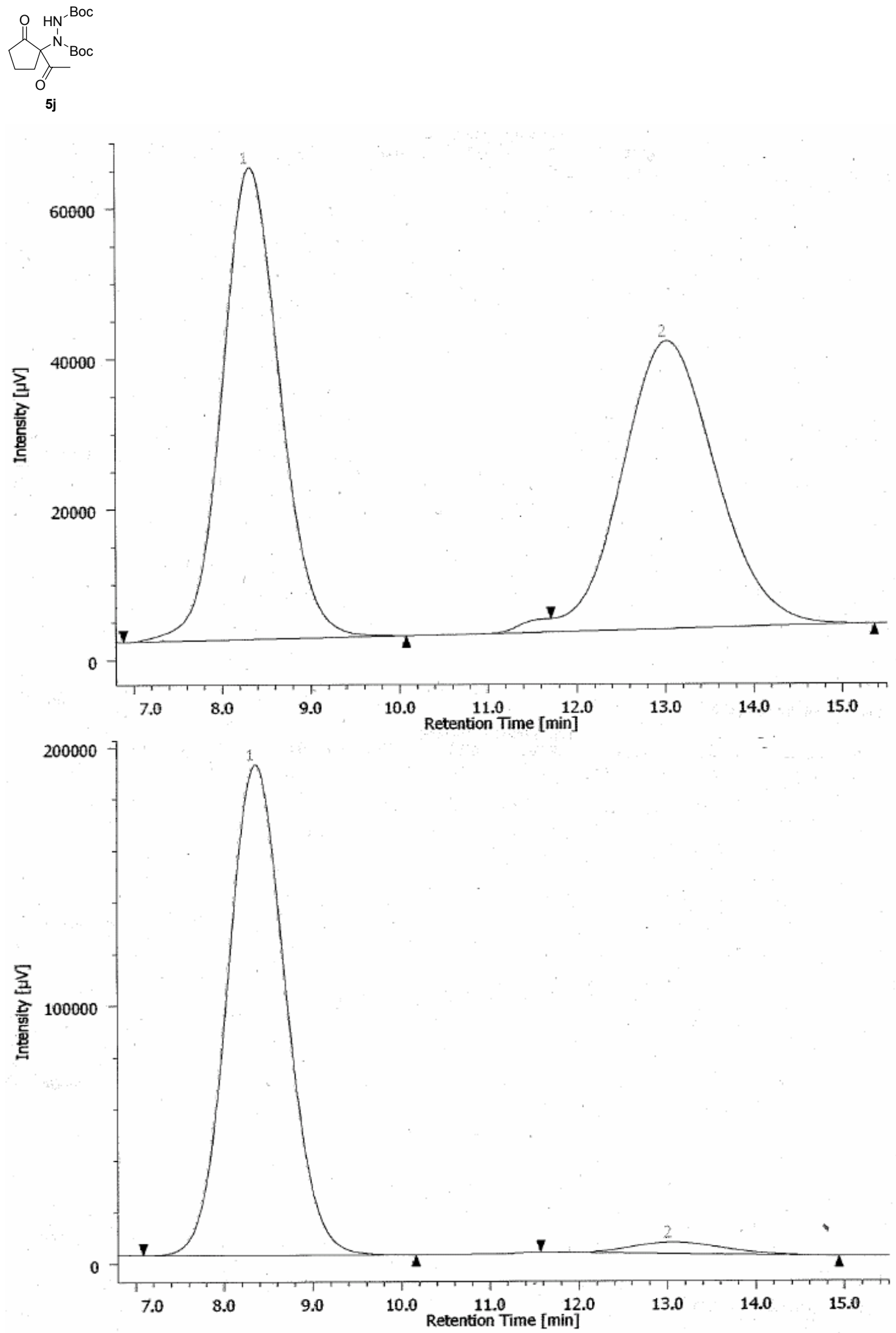